\title{
Maximizing the value of science: no news can be good news
}

\author{
Michael Mandler* \\ Royal Holloway College, University of London
}

This version: May 2011

\begin{abstract}
When scientists choose research projects with the highest expected value an externality can appear; slight variations on existing work will be selected in preference to new lines of research that would ultimately generate more value. New research enjoys two advantages: it is riskier and hence more likely to lead to high-value spin-off projects and it can generate more spin-off projects. Less communication among scientists can mitigate the externality, as can the presence of scientists who are risk-takers and secretive. When scientists maximize citations rather than expected value, the externality can but need not be alleviated.
\end{abstract}

JEL codes: D62, D83, H41, Z19

Keywords: science, innovation, externalities, citations, trees, martingales

*Address: Department of Economics, Royal Holloway College, University of London, Egham, Surrey, TW20 0EX, UK. Email: m.mandler@rhul.ac.uk. 


\section{Introduction}

Thanks to the internet, scientists nowadays learn the results of their peers' research with little delay. This development might appear to be unambiguously good: armed with the knowledge of what research has already been undertaken and how successful it has turned out to be, other scientists can build on past successes and make better decisions about which projects to pursue. An externality, however, can overturn this scenario. When a researcher does not know what projects others are pursuing - or has only vague information - he or she may be forced into initiating a new and riskier line of research that has greater upside potential or that has a greater potential to generate spin-off projects. Although work on a new line of research will likely have smaller expected value than work in existing fields, if the new research turns out to be successful then other scientists can undertake a stream of related projects that will generate enough value to outweigh the costs of experimentation.

Two mechanisms are at work. First, while projects in existing fields may have higher expected value they will normally be less risky. But risk is an advantage. If a new field turns out to have high value then related projects will be available that also have high value while if the new field is of poor quality it can simply be abandoned. Since for these follower projects there is a potential upside gain but no downside loss, greater dispersion brings a net benefit. Second, even if new fields are no riskier they may generate more streams of successor projects. Consequently in the event that a new field has high value but one of its streams of successors turns out to be a failure, there will be other streams to turn to. To incorporate this second 'fallback' effect, we view new fields as trees whose branches represent spin-off projects. When either mechanism is in play, investment in a new field will be socially beneficial even though it will in expectation incur a short-term loss of research value. In at least some cases, therefore, impeding the flow of knowledge can lead to a social gain: scientists might experiment with new approaches when they are intellectually isolated, thus ultimately increasing the flow of research value.

The above story is motivated in part by a chapter in the history of particle physics. By

the late 1960's, much of the particle physics community had rejected quantum field theory and instead followed the latest fashion, the 'bootstrap model.' Some heterogenous pockets 
remained out of the loop, however, especially those distant from the epicenter of bootstrap research on the west coast of the U.S. (Ne'eman (1982)). In particular, a group of physicists in the Soviet Union - an academic 'island' in the words of Polyakov (1997) - continued to pursue a theory of gauge fields that would eventually be harnessed to describe the three fundamental forces in today's standard model of particle physics. With the triumph of the standard model, the bootstrap model faded away. The moral of the story is that it can be valuable to have several scientific schools following different lines of research in ignorance of each other's work. ${ }^{1}$ When in contrast everyone knows exactly what every other researcher is doing and they all judge the expected value of research in the same way, then the pursuit of the highest-value projects will lead individuals to herd, with all researchers pursuing similar lines of attack.

The history of science is often portrayed as a struggle between a few heroic paradigm shifters and the larger crowd that dutifully pursues Kuhnian normal science; progress is slow in this account because of the shortage of brave geniuses. This paper will retain the Kuhn (1962) distinction between innovative and normal science but argue against the 'hero' explanation of the divide. The emphasis here will be on incentives: although it can be socially suboptimal to work in existing fields, the scientists who make this decision are not automatons blindly chasing down the routine details left behind by smarter pioneers, they pick projects that will in fact maximize the value of their own research. An echo of the 'hero' model will remain, however, in that scientists with a taste for risk and secrecy can mitigate the externality that afflicts research decisions and increase the flow of research value over time.

It might seem that if scientists maximize their citations rather than the value of their research then the externality will vanish; citation pursuers will get credit from all of the offshoots of the fields they initiate. It turns out that the pursuit of citations can but does not always encourage investment in new fields. Since a new field must show sufficiently high value for it to garner citations, the same forces that discourage value-maximizing scientists from

\footnotetext{
${ }^{1}$ Ne'eman (1982) emphasizes that the development of gauge theory was spurred by the independent investigations of heterogeneous schools of physicists. Polyakov (1997) sounds a similar theme. The above account draws on the ideas of the physicist Rafael Sorkin who has stressed the advantages of limited communication among schools. For histories of this episode, consistent with the interpretation we have given, see Hoddeson et al. (1997) and 't Hooft (1999).
} 
innovating apply to citation-maximizers as well. Depending on the parameters, citations can also lead to an overshooting where excessively many new fields are initiated.

Among the many forces guiding scientific research that this paper neglects, the most prominent is the role of journal editors and referees. But we will at least see (in section 7) that the refereeing process can blunt the citation incentive to initiate new fields and that referees rather than editors present the larger incentive problem.

In the equilibria of our model scientists will herd into the field with the highest value projects, but the logic that drives this clustering is different from the herds of Banerjee (1992) and Bikhchandani et al. (1992). It is not the case in our model that some scientists have better information and that other scientists for this reason mimic their decisions. We take the opposite tack of assuming that scientists share a common pool of information; herding is instead a consequence of the correct (symmetric information) pursuit of self-interest. A closer match is the 'learning by doing' model of Jovanovic \& Nyarko (1996) where an agent can achieve long-run productivity growth only if his momentary expertise in the technology he knows best is not so great that the agent declines to experiment with new technologies. While a similar lesson holds here - access today to higher quality projects can bring about a long-term loss - the mechanics are different. In our model, riskiness is indispensable if new fields are to deliver a benefit to society whereas in $\mathrm{J} \& \mathrm{~N}$ riskiness produces no direct social gain. New technologies in $\mathrm{J} \& \mathrm{~N}$ instead derive their advantage from their greater long-run productivity; in our model any specific new field is a poor prospect but it is optimal to sample new fields since they can be dropped whenever they turn out to have low value.

Perhaps the work closest to the present paper is Hong \& Page (2004) who argue that a population of agents who use a diverse set of problem-solving procedures can outperform a population of high-ability agents. Though the setting is different, the best-performing agents in Hong \& Page suffer from the drawback that they all pursue the same solution to a problem, comparably to the scientists in our model who cluster in the same field.

Finally we mention a different inefficiency that scientific research can generate: the duplication of effort in the race to be the first to make a discovery, which amounts to another type of herding. See Dasgupta \& David (1994) and Dasgupta \& Maskin (1987). In the present paper, we will assume duplication away - multiple scientists will never undertake the same 
project and the success of a project will only add to the expected value of its neighbors not due to a belief that duplication is unimportant but to clarify that the externality under discussion works by a different path. The role of secrecy in science is also discussed in Dasgupta-David but they consider its negative side not its potential to remedy inefficiency that we discuss in section 5.2 .

\section{Projects, fields, and trees of knowledge}

Scientific research will proceed via a sequence of projects that are organized into fields. A project is a completed work of research ready for publication while a field is an innovation that makes a new set of projects possible. A new field's innovation can be theoretical and does not have to uncover new phenomena; if it did not carry so much freight, 'paradigm' might be a better expression than 'field'.

A new field may be initiated at any time, but within a field progress is cumulative and therefore the projects that are currently available are determined by the projects undertaken in the past. We model this relationship by assuming that each field $f$ is a tree: there is a root project, labeled $(0, f)$, which determines a set of successor projects or branches, each of which in turn determines a set of successor projects, and so on. Except for root projects, no project can be undertaken before its immediate predecessor has been.

For the issues we pursue, it will be enough to consider only trees where every non-root project has just one successor: the root project initiates a field and leaves in its wake a set of successors, each of which has a single successor, and so on. A tree for a field can therefore be characterized by the integer number of successors, $\beta$, to the root project and accordingly is called a $\beta$-tree. We assume there is a $\beta>0$ such that every field in the model is a $\beta$-tree. See Figure 1 for two sample trees. We could generalize considerably and even introduce uncertainty about tree structure: what is important is that root projects are expected to have more branches (successor projects) than nonroot projects.

Each project in a field $f$ is identified by the triple $(b, i, f)$, where $b$ indexes the branch that

leads to $(b, i, f)$ and $i$ is the number of steps between the root project and $(b, i, f)$. We use $(b, 0, f)$ as well as $(0, f)$ to denote the root project of a field $f$. So for any project $(b, i, f)$, 


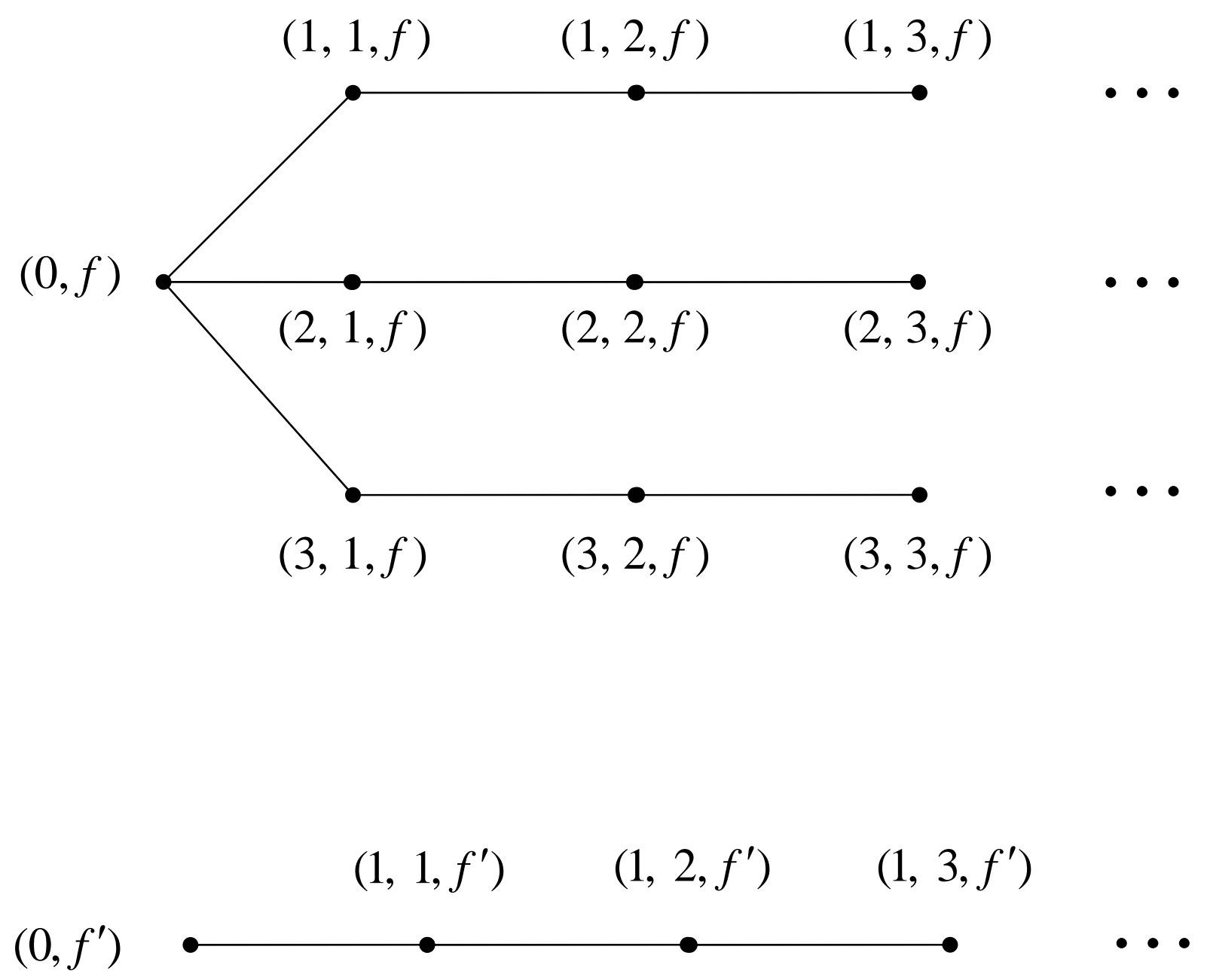

Figure 1: a 3-tree and a 1-tree 
its immediate successor is $(b, i+1, f)$ and (if it is not a root) its immediate predecessor is $(b, i-1, f)$. A generic project, either root or follower, is labeled $\pi$.

We consider a finite sequence of scientists $1, \ldots, T$ each of which undertakes a single project, where scientist $t$ chooses at date $t$. The projects that are feasible at $t$ are the root projects of fields that have not yet been initiated and the projects that have not yet been undertaken but that are the immediate successors of projects that have been. ${ }^{2}$

Each project $\pi$ has a value $v(\pi)$, a real number that indicates the project's worth and that should be interpreted as the knowledge or value added by $\pi$ given the value already generated by $\pi$ 's predecessors in its field. The values of projects are uncertain and so a state $\omega$ will specify the value of all possible projects. $P(\cdot)$ will indicate the probabilities of sets of states.

Agents do not know $v(\pi)$ before $\pi$ is undertaken. But once $\pi$ is undertaken by scientist $t$ then $v(\pi)$ is revealed before scientist $t+1$ decides on a project and is remembered ever after.

Let a history be a $h_{t}=\left(\pi_{1}, \ldots, \pi_{t} ; v\left(\pi_{1}\right), \ldots, v\left(\pi_{t}\right)\right)$ where $\pi_{i}$ indicates the project adopted in period $i$. For each $h_{t}$, we require that if $(b, i, f)$ is in $h_{t}$ - that is, $(b, i, f)$ is one of the first $t$ coordinates of $h_{t}-$ and $i \geq 1$ then $(b, i-1, f)$ is in $h_{t}$, reflecting the fact the projects in a branch must be undertaken in sequence. In terms of histories, a project $(b, i, f)$ is feasible following $h_{t}$ if $(b, i, f)$ is not in $h_{t}$ and, when $i \geq 1,(b, i-1, f)$ is in $h_{t}$.

A scientist's decision about which project to undertake turns on the expected values of the projects that are available. We assume that (1) ex ante any two root projects have the same expected value and (2) the value of a project is positively correlated with the value of its immediate predecessor. The upshot of these assumptions is that when a project $\pi$ in an existing field is undertaken and revealed to have positive value then each of $\pi$ 's successors will have greater expected value than any root project $\pi^{\prime}$ of a new field or any of the successors of $\pi^{\prime}$. New fields will then be an unattractive source of projects.

In stating (1) precisely, we normalize the common ex ante expected value of new fields to 0:

\footnotetext{
${ }^{2}$ To ensure that each scientist has the option of initiating a new field, we assume that there are at least $T$ fields.
} 
Assumption 1 (common expected values for fields) For all fields $f$ and all histories $h_{t}$ such that $(0, f)$ is not in $h_{t}$, the conditional expectation of $v(0, f)$ given $h_{t}$ equals 0 : $E\left(v(0, f) \mid h_{t}\right)=0 .^{3}$

The reader should not assume that 0 or negative value projects are worthless or destructive. Even a line of research that fails on its own terms imparts knowledge, if only the knowledge that a candidate theory is false or, in technological applications, that an invention does not work.

For (2), we assume that the expected value of a project equals some common fraction or multiple of the value of the project's predecessor. Given a history $h_{t}$, let a leading project of $h_{t}$ be a project $(b, i, f)$ in $h_{t}$ such that $(b, i+1, f)$ is not in $h_{t}$.

Assumption 2 (correlated values) There is a number $\delta>0$ such that, for any history $h_{t}$ and any leading project $(b, i, f)$ of $h_{t}$,

$$
\begin{aligned}
& v(b, i, f) \geq 0 \Rightarrow E\left(v(b, i+1, f) \mid h_{t}\right)=\delta v(b, i, f), \\
& v(b, i, f)<0 \Rightarrow E\left(v(b, i+1, f) \mid h_{t}\right)<0 .
\end{aligned}
$$

Assumption 2 implies that for as long as the values of projects in a single branch of a field remain nonnegative they follow a martingale, supermartingale, or submartingale as $\delta=1$, $\delta \leq 1$, or $\delta \geq 1$.

When $\delta<1$, the returns to pursuing a productive line of research progressively diminish as the research is pushed further. The expected value of a project with positive value then reverts to the mean, which under our normalization is 0. Assumption 2 allows negative value projects to not revert to the mean even when $\delta<1$; in particular a field can fail with no chance of recovery as when its capacity to yield further insight is completely exhausted. Our structural assumption that any project has a potential successor therefore has no bite: the endless supply of projects in a field can all be nearly worthless (have a highly negative value).

The requirement in Assumption 2 that $\delta$ is fixed across fields and projects could be

\footnotetext{
${ }^{3}$ Here and subsequently $E(x)$ will be the expectation of the random variable $x$ and $E(x \mid A)$ will be the conditional expectation of $x$ given the event $A$. We define $h_{t}$ as an event explicitly in Appendix A.
} 
substantially weakened. Its role is provide a tractable parameter that determines whether project values regress to the mean. Notice however that the requirement rules out correlation of expected values across branches of a single field: once the value of some root project $(0, f)$ has been discovered then every immediate successor of $(0, f)$ that has not yet been undertaken has the conditional expected value $\delta v(0, f)$, regardless of what $v(b, 1, f)$ 's have been revealed. But the correlations that are ruled out could go either way. For example, suppose $(0, f)$ is a theoretical innovation and that each immediate successor amounts to an application of this original insight. It could well be that when one application, say $(b, 1, f)$, is unusually successful - that is, $v(b, 1, f)>\delta v(0, f)$ - then one should infer that the theory works in the real world and hence that a second application $\left(b^{\prime}, 1, f\right)$ will be successful too. Equally it could be that all of the applications are minor variations on a theme; hence only the first application delivers a substantial incremental insight, with the remainder delivering nearly the same message. In the first case $v(b, 1, f)$ and $v\left(b^{\prime}, 1, f\right)$ will be positively correlated and in the second they will be negatively correlated. Assumption 2 steers a middle course.

Given Assumptions 1 and 2, the law of iterated expectation implies that conditional on a history $h_{t}$ any project in a field that has not yet been initiated in $h_{t}$ has 0 expected value (assuming that a branch of a field terminates at the point where projects in that branch first hit a negative value). In contrast any successor of a leading project $\pi$ with $v(\pi)>0$ has positive expected value. Not surprisingly, agents whenever possible will take up projects in fields with positive-value leading projects.

Although we will not exploit all its possibilities, the above model of scientific innovation is rich enough to cover fields that are broad (the number of branches $\beta$ is large) but not deep ( $\delta$ is small), as for example when a scientific theory generates many applications but no further conceptual developments. Conversely, a deep but narrow field could have a single branch $(\beta=1)$ whose projects have increasing expected value ( $\delta$ is large).

The model intentionally mimics Kuznets's celebrated (1930) account of technological innovation and thus indirectly draws on Schumpeter (1911) too. In Kuznets, every sector in an economy eventually falls victim to the law of diminishing returns to technical innovation. But though each sector is doomed to eventual stagnation, the economy as a whole can grow robustly due to the neverending supply of new 'leading' sectors. A corresponding pattern 
appears in the present model. When $\delta<1$, any successful field whose current projects have high value will eventually run through its stock of great ideas. But as we will see the presence of new fields can lead to an endless stream of projects with positive expected value, despite the fact that any one new field ex ante generates projects of 0 expected value. Although we will not dwell on a technological interpretation, the fields in our model can be understood as major innovations, projects as lesser adaptations, and the diminishing accumulation of value that can occur when $\delta<1$ as a fall in the rate of economic growth.

\section{Plan and equilibrium}

A plan consists of $T$ functions $a=\left(a_{1}, \ldots, a_{T}\right)$ where each $a_{t}$ assigns to each history $h_{t-1}$ a project that is feasible following $h_{t-1}$. Given the probabilities of states, a plan defines a probability that any given set of projects is undertaken and a total expected value for the scientific research undertaken from 1 to $T$. The exact definitions of these probabilities and expectations are a tedious and so we segregate them into Appendix A.

We assume initially that each scientist maximizes the expected value of the project that he/she undertakes.

Definition 1 Given the history $h_{t-1}$, a project $\pi$ is an equilibrium choice (at $t$ ) if

- $\pi$ is feasible following $h_{t-1}$,

- $E\left(v(\pi) \mid h_{t-1}\right) \geq E\left(v\left(\pi^{\prime}\right) \mid h_{t-1}\right)$ for all $\pi^{\prime}$ that are feasible following $h_{t-1}$.

An equilibrium is a plan $\left(a_{1}, \ldots, a_{T}\right)$ such that each $a_{t}$ assigns an equilibrium choice to each history $h_{t-1}$.

Assumptions 1-2 imply that to identify an equilibrium choice when facing $h_{t-1}$ the scientist at $t$ need only look at the leading projects of $h_{t-1}$, choose the successor of a leading project with the highest value if that value is positive, and otherwise initiate an arbitrary new field. 


\subsection{A random walk illustration}

To see how an equilibrium proceeds and illustrate the Kuznets/Schumpeter implications of the model, suppose the value of projects in each field follows a standard random walk: any root project either has value 1 or -1 , each with probability $\frac{1}{2}$, while any other project has the value of its immediate predecessor plus or minus 1 , each with probability $\frac{1}{2}$. So, for each $h_{t}$,

$$
P\left(v(0, f)=1 \mid h_{t}\right)=P\left(v(0, f)=-1 \mid h_{t}\right)=\frac{1}{2}
$$

if $(0, f)$ is not in $h_{t}$ and

$$
P\left(v(b, i, f)=v(b, i-1, f)+1 \mid h_{t}\right)=P\left(v(b, i, f)=v(b, i-1, f)-1 \mid h_{t}\right)=\frac{1}{2}
$$

if $(b, i-1, f)$ is a leading project in $h_{t}$.

Since fields are all ex ante identical, let the date 1 scientist initiate an arbitrary field $f$. Suppose fields have just one branch $(\beta=1)$ and - in contrast to what happens in equilibrium - that all scientists at dates $t>1$ simply undertake the sole available project in $f$ that is currently available. Since there is then no further initiation of new fields after date 1 the sequence of realized values will form a standard random walk and hence the expectation (at date 0 ) of the value of research achieved at each date $t$ will be 0 .

An equilibrium performs better than this benchmark. Still assuming that each field has a single branch, the scientists at dates $t>1$ will undertake the immediate successor of the project $\pi_{t-1}$ undertaken at $t-1$ if $v\left(\pi_{t-1}\right)>0$ and will initiate a new field if $v\left(\pi_{t-1}\right)<0$. (If $v\left(\pi_{t-1}\right)=0$ the date $t$ scientist can either select $\pi_{t-1}$ 's successor or initiate; assume for concreteness that $t$ chooses $\pi_{t-1}$ 's successor.) This strategy is the mirror image of the classic gambling strategy of continuing to place bets until one's stake hits positive territory: scientists in equilibrium pursue a field until its value turns negative. Now in any equilibrium the expected value of the terminal project of a field $f$ - the project in $f$ that is chosen immediately prior to the initiation of another field or at period $T$ - will equal $0 .{ }^{4}$ In Figure 2 , the values of terminal projects when $T=4$ are recorded at the terminal nodes; each

\footnotetext{
${ }^{4}$ Since the values of projects in a single field form a martingale, this conclusion follows from the Doob stopping theorem (see Williams (1991)).
} 


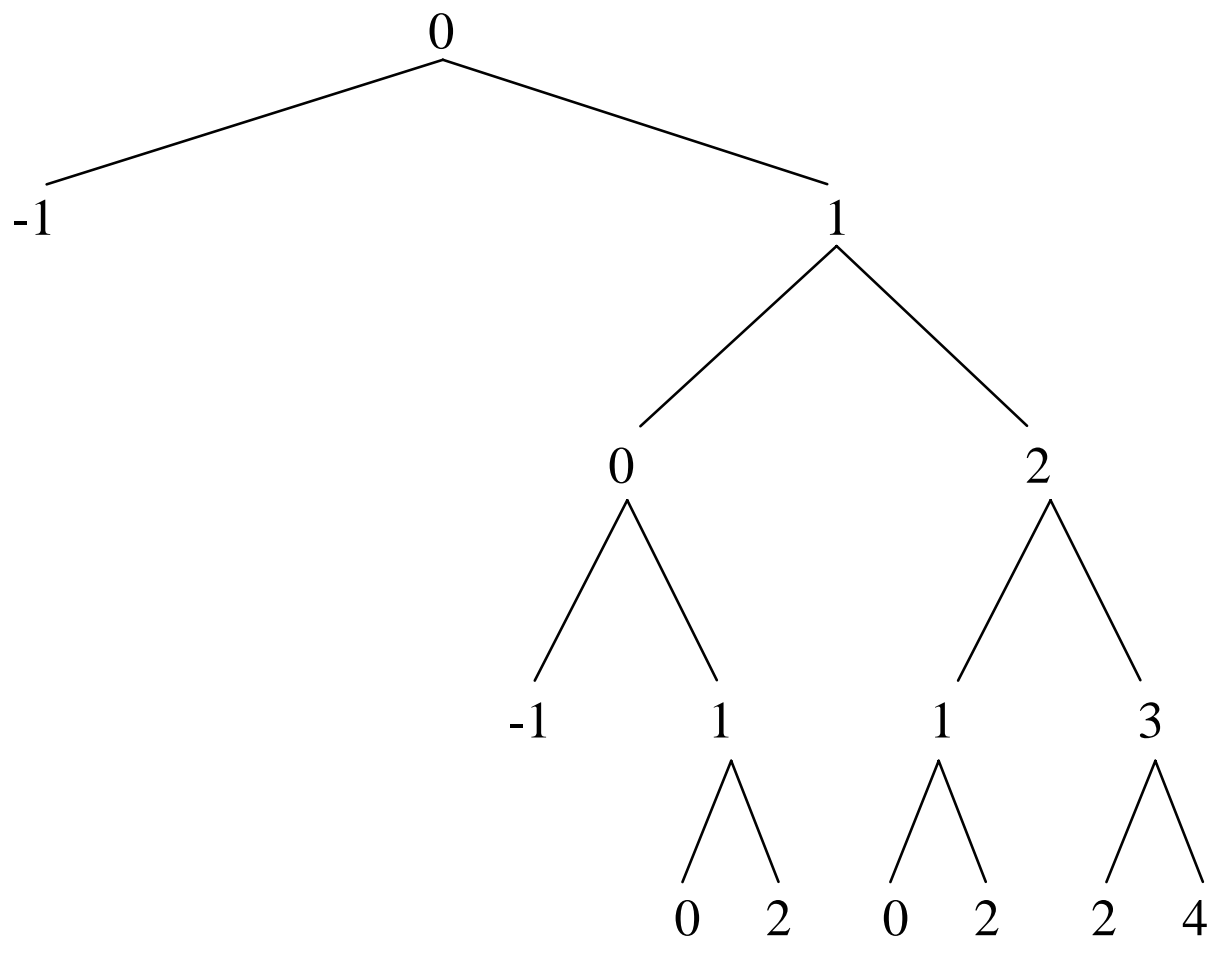

Figure 2: possible project values in a single field

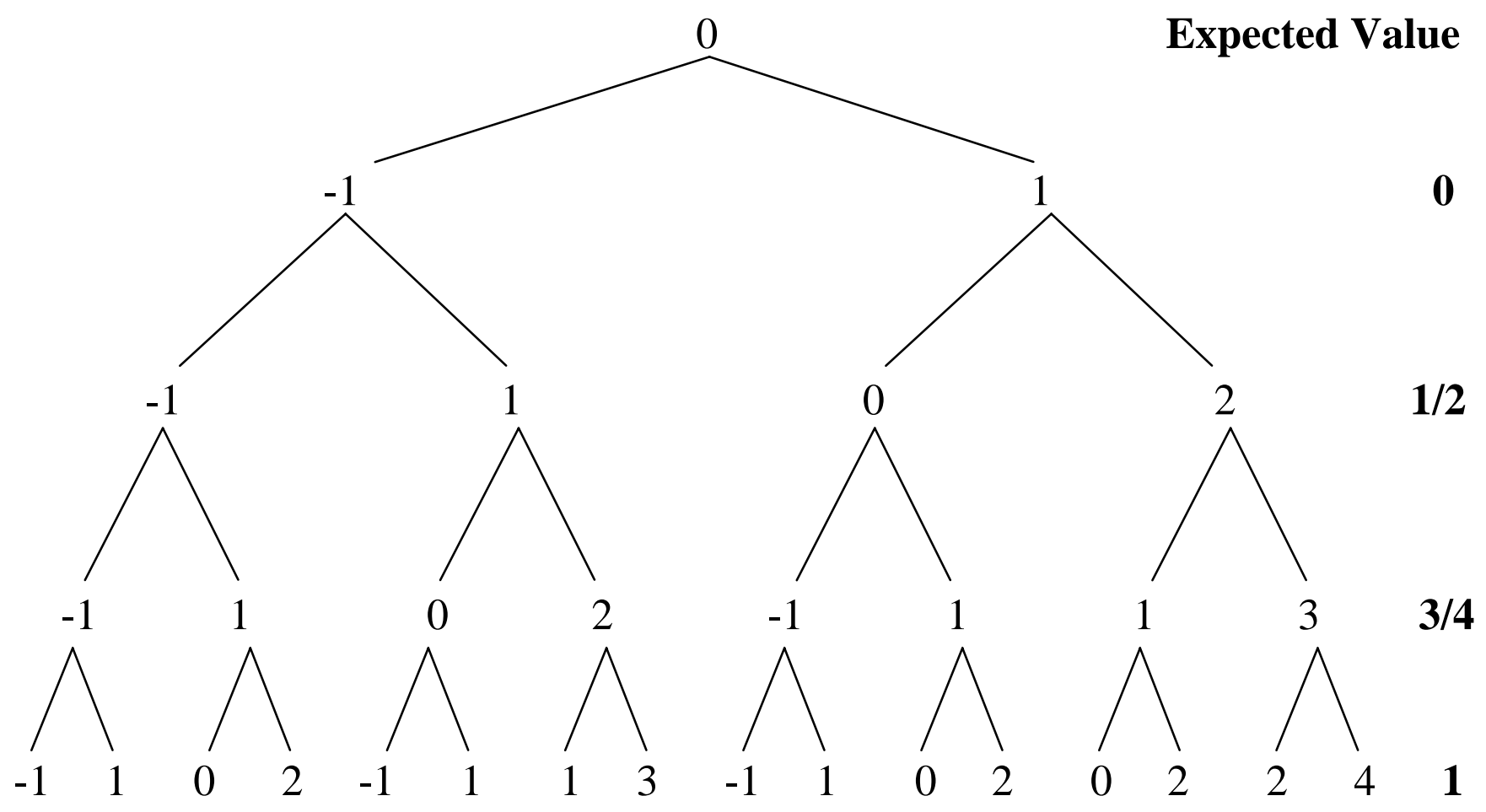

Figure 3: possible project values with new field initiation 
fork in the figure represents the two possible values that a project might have, not a set of successors to a project (we have set $\beta=1$ ). Since in equilibrium a new field is initiated when an existing field hits the value -1 , a new field whose expected value in the subsequent period is 0 must be inserted at those points, as pictured in Figure 3. This increase from an expected value of -1 to an expected value of 0 implies that the expected value of research in each period $t>1$ must be strictly positive; in the first four periods of any equilibrium, the expected values of research turn out to be $0, \frac{1}{2}, \frac{3}{4}, 1$.

The curiosity of the random-walk model is that the expected value of the terminal project in any newly initiated field remains exactly equal to 0 . It is the fact that an equilibrium will initiate a new field whenever the projects in an existing field turn negative that leads the stream of expected values to be positive overall.

When the number of branches $\beta$ is greater than 1 , equilibria perform better. If the first agent selects field $f$ and $v(0, f)=1$, and the scientists who follow select projects from, say, branch 1 then, once $v(1, i, f)$ hits 0 (as it will eventually with probability 1 ), subsequent scientists will undertake projects in another branch of $f$. The values in this branch have expected value 1 rather than the expected value of 0 that would obtain in a new field.

But equilibria do not fully exploit the opportunities offered by fields with $\beta>1$. If a scientist has a choice between a project with expected value 0 in an existing field and initiation of a new field, he will be indifferent between the options. But initiation of a new field has the upper hand from society's point of view. If the new field $f$ turns out to have the high realization $v(0, f)=1$ and the first successor of $f$ undertaken turns out to have value 0 - a low realization given that $v(0, f)=1$ - then the next scientist can adopt another of $(0, f)$ 's immediate successors, which will have expected value 1 . When the same chain of realizations occurs with a 0-expected-value project in an existing field - first a high realization then a low realization - there is no 'fall-back' option available with expected value greater than 0 . This multi-branch externality is a key advantage of new fields and we will consider it in detail in section 4.2. The present version of the externality is not very impressive since it disappears if scientists choose to initiate a new field rather than select an existing-field project with expected value 0 . But the externality becomes robust when we leave the narrow confines of this random walk example. 
It so happens that in any equilibrium for this example the expected value of research achieved at date $t$ increases without bound as $t$ increases, a consequence of the fact that the value of projects in a field does not diminish in expectation as more are undertaken. If we replace our implicit assumption that $\delta=1$ with $\delta<1$ and use

$$
P\left(v(b, i, f)=\delta v(b, i-1, f)+1 \mid h_{t}\right)=P\left(v(b, i, f)=\delta v(b, i-1, f)-1 \mid h_{t}\right)=\frac{1}{2}
$$

rather than (3.1), then the expected value of research per period will be bounded above. But equilibria still achieve a stream of positive expected values: even in the presence of diminishing returns in every field, the expected value of research in equilibrium will be strictly positive at each $t>1$, comparably to the Kuznets growth model.

\section{The externality}

Society's interest lies in maximizing the total expected value of research, defined formally in Appendix A. An equilibrium of self-interested scientists, each maximizing the value of his or her own project, can fail to achieve this goal; in particular, the initiation of new fields can fall short of the optimal level.

If some leading project $\pi$ in an existing field has positive value then every project in any new field has smaller expected value than any successor of $\pi$ (see section 2). If science were risk-free, that would be the end of the story: society will be better off if scientists undertake the successors of the positive-value projects in existing fields and that is what self-seeking scientists will do. It is indispensable therefore that new fields are risky if there is to be a social gain to investing in them when positive-value fields are available. The next subsection considers the pure risk advantage of new fields. Later, in section 5.1, we compare the research value achieved by arbitrary projects with different risk levels. In subsection 4.2 , we turn to the second benefit of new fields: when a new field has multiple branches and one branch turns out to be a failure, there are fall-back branches to turn to. 


\subsection{The certain-continuation model}

To isolate the benefit of the greater riskiness of new fields compared to projects that continue old fields, we begin by going to the extreme of assuming that the projects that continue old fields display no variability at all. Specifically, suppose for any history $h_{t}$ that if $(b, i, f)$ is a leading project of $h_{t}$ and $v(b, i, f)>0$ then $v(b, i+1, f)$ is certain to equal $\delta v(b, i, f)$,

$$
P\left(v(b, i+1, f)=\delta v(b, i, f) \mid h_{t}\right)=1
$$

The value of root projects remains uncertain; we suppose that the value of each root project is governed by the same density $g .{ }^{5} \quad$ When these assumptions hold, we have a certaincontinuation model. We assume that each distribution in this paper that admits a density has support equal to an interval.

Consider the scientist who chooses at $t$ and faces history $h_{t-1}$. Let $\delta$ equal 1 , which in the certain-continuation model means that every project in the same field has a common value. Evidently if scientist $t$ is going to choose a project in a field that has already been initiated then he should choose that field that has been revealed to have the highest value, and in this decision there is no divergence between the scientist's and society's interests. As for the initiation decision, in equilibrium scientist $t$ will choose a project from an existing field if some existing field has been revealed to have projects with positive value. To maximize society's knowledge, however, scientist $t$ should in some of these cases initiate a new field instead: if a new field has a high value then society can reap a continuing gain while if it has low value then other scientists can return to existing fields or initiate again. Let $\bar{v}$ be the maximum of the values that have been revealed in already-initiated fields. Then, since the expected value of a new field is 0 , the cost of initiating a new field at $t$ is just $\bar{v}$. But the gain from $t+1$ onwards, assuming there are no further new field initiations, is given by

$$
(T-t) \int_{\bar{v}}^{\infty}(v-\bar{v}) g(v) d v
$$

the number of periods that remain following $t$ multiplied by the expected gain in excess of

\footnotetext{
${ }^{5}$ That is, if the history $h_{t}$ is such that $(0, f)$ is not in $h_{t}$ then $P\left(v(0, f) \in A \mid h_{t}\right)=\int_{A} g(v) d v$.
} 
$\bar{v}$ if the new field indeed has a value that exceeds $\bar{v}^{6}$ The above measure of the gain from initiation in fact holds when we allow for repeated initiations and, with a slight adjustment, can cover the $\delta>1$ case too. The $\delta<1$ case does not lead to a clean formula since it can be optimal to switch among existing fields as their projects deteriorate in value.

We will say that a plan a underinvests in new fields following history $h_{t-1}$ if $a$ does not initiate a new field following $h_{t-1}$ and there is an alternative plan $a^{\prime}$ that is identical to $a$ through $t-1$, that does initiate a new field following $h_{t-1}$, and that increases the total expected value of research conditional on $h_{t-1}$. See Appendix A for more details.

Proposition 1 Suppose $\delta \geq 1$. If in an equilibrium of the certain-continuation model $\bar{v}>0$ is the maximum expected value of projects that are feasible following history $h_{t-1}$, then the equilibrium underinvests in new fields following $h_{t-1}$ if and only if

$$
\sum_{i=1}^{T-t} \delta^{i} \int_{\bar{v}}^{\infty}(v-\bar{v}) g(v) d v>\bar{v}
$$

Proof. The value of research from $t$ through $T$ achieved by an equilibrium $\left(a_{1}, \ldots, a_{T}\right)$ conditional on $h_{t-1}$ equals $\sum_{i=0}^{T-t} \delta^{i} \bar{v}=\bar{v}+\sum_{i=1}^{T-t} \delta^{i}\left[\int_{\bar{v}}^{\infty} \bar{v} g(v) d v+\int_{-\infty}^{\bar{v}} \bar{v} g(v) d v\right]$ while the sum of the expected values of research from $t$ through $T$ conditional on $h_{t-1}$ if a new field $f^{\prime}$ is initiated at $t$ but where $a_{j}$ determines the choice of action for $j>t$ equals $E\left(v\left(0, f^{\prime}\right) \mid h_{t-1}\right)+$ $\sum_{i=1}^{T-t} \delta^{i} \int_{\bar{v}}^{\infty} v g(v) d v+\sum_{i=1}^{T-t} \delta^{i} \int_{-\infty}^{\bar{v}} \bar{v} g(v) d v$. Since $E\left(v\left(0, f^{\prime}\right) \mid h_{t-1}\right)=0$, the latter strategy strictly increases the sum of the expected value of research if (4.1) obtains. If we allow for the possibility of initiating another field after $t+1$, (4.1) will still be sufficient for new-field initiation at $t$ to be a strict expected improvement since we can always forego the option of initiating again after $t$.

To argue for necessity, suppose that $\sum_{i=1}^{T-t} \delta^{i} \int_{\bar{v}}^{\infty}(v-\bar{v}) g(v) d v \leq \bar{v}$. Then, for initiation of a new field at $t$ to lead to a strict increase in the sum of the expected values of research through $T$, there must be a plan $a^{\prime}$ identical to $a$ through date $t$ and a state $\omega$ such that (i) $a^{\prime}$ initiates a new field at some date $\tau>t$ following the history $h_{\tau-1}$ to which $\omega$ leads, and (ii) the conditional expected value of research from $\tau$ through $T$, given $h_{\tau-1}$, is greater

\footnotetext{
${ }^{6}$ The above expression is comparable to the formula for the returns to search in the McCall (1970) model of the labor market.
} 
with $a^{\prime}$ than with $a$. But consider the last date $\tau^{\prime}$ at which $a^{\prime}$ initiates a new field given $\omega$. Since the left hand side of (4.1) is strictly decreasing in $t$ and since $\bar{v}$ cannot decrease, this last initiation of a new field must lead to a decrease in the conditional expected value of research from $\tau^{\prime}$ through $T$. Hence there can be no state where initiation at $\tau>t$ leads to a strict improvement, conditional on $h_{\tau-1}$, and so initiation of a new field at $t$ cannot lead to a strict increase in the sum of the expected values of research.

When $\delta=1$, it is easy to use Proposition 1 to show that when $T$ is large enough then an equilibrium will be sure to underinvest in new fields. To allow $T$ to vary, we consider equilibrium sequences $\left(a_{1}, \ldots, a_{t}, \ldots\right)$ (see Appendix A). Since it is a probability 0 event for a root project to have value exactly equal to 0 , an equilibrium sequence will, with probability 1 , eventually undertake a root project $(0, f)$ with strictly positive value $\bar{v}$. A glance at inequality (4.1) shows that if $f$ is initiated at $t-1$ then, since $\sum_{i=1}^{T-t} \delta^{i}$ increases without bound as $T$ increases, (4.1) must be satisfied when $T$ is sufficiently large. So then the equilibrium sequence underinvests at $t$. When $\delta<1$ a slightly different argument is needed: in equilibrium only a single field is pursued even when its projects have negligible but positive value whereas given enough time unboundedly great value can be achieved by initiating a new field at any date where the value of projects in existing fields has dropped far enough. See Appendix B, which contains all proofs omitted from the text.

Proposition 2 Suppose the certain-continuation model satisfies $\delta \leq 1$. Then with probability 1 any equilibrium sequence will underinvest in new fields at some date.

\subsection{The fixed-dispersion model}

The certain-continuation model shows that one benefit of a new field is that it may have a larger probability of containing high value projects than an already initiated field has. An existing field could contain only low-value projects for many reasons: perhaps it never had any high-value projects to begin with, or perhaps the field has 'played out' which is sure to occur eventually when $\delta \leq 1$. But a greater chance of containing high-value projects is not the only advantage of new fields. To neutralize the risk benefit of new fields, we will now assume any two feasible projects have the same distribution of values after correcting for 
any difference in their means. Formally, there will be a density $g$ such that if a project $\pi$ is feasible following history $h_{t}$ and $E\left(v(\pi) \mid h_{t}\right)=e$ then the conditional distribution of $v(\pi)$ given $h_{t}$ is governed by the density $g_{e}$ defined by $g_{e}(v)=g(v-e){ }^{7}$ When this assumption holds, we say that fixed dispersion is satisfied.

Under fixed dispersion, the distribution of values of a root project of a new field has a less advantageous upper tail than the upper tail of any project in an existing field with positive expected value: for any $r>0$, any $h_{t}$, any feasible $\pi$ such that $E\left(v(\pi) \mid h_{t}\right)>0$, and any $f^{\prime}$ that has not yet been initiated,

$$
P\left(v(\pi)>r \mid h_{t}\right)>P\left(v\left(0, f^{\prime}\right)>r \mid h_{t}\right) .
$$

Yet under fixed dispersion it remains possible that an equilibrium underinvests in new fields. It is here that the tree structure of fields comes into play. When fields have more than one branch, the initiation of a new field has a signal advantage: if the root project $(0, f)$ has high value but the first successor of $(0, f)$ undertaken turns out to have an unexpectedly low value then other scientists can pursue the higher expected-value projects available in the remaining $\beta-1$ branches of $f$. In a single branch of an existing field or in a 1-tree, in contrast, there are no such fall-back options. Consequently, when $\beta \geq 2$ and the expected values of projects in existing fields are sufficiently low it will be worthwhile from society's point of view to sacrifice those small expected values to gain the fall-back advantages offered by a new field.

Cutoff Lemma If fixed dispersion holds then the following two conditions are equivalent: (1) there exists a cutoff $c>0$ for the value of projects such that, for all $t \leq T-2$ and all $h_{t-1}$ such that the highest value leading project has a value in $(0, c)$, any equilibrium plan underinvests in new fields following $h_{t-1}$,

(2) the number of branches in each field is greater than or equal to 2.

Since for any $c>0$ there is a positive probability that the project undertaken at date 1 turns out to have a value in $(0, c)$, the cutoff lemma implies that underinvestment at date

\footnotetext{
${ }^{7}$ So $\int_{-\infty}^{\infty} v g_{e}(v) d v=e$ and, for any measurable $A, P\left(v(\pi) \in A \mid h_{t}\right)=\int_{A} g_{e}(v) d v$. We assume that the interval support assumption given in section 4.1 continues to hold.
} 
2 will occur with positive probability if fields have at least two branches and there are at least two additional periods following date 2 . The proof of the cutoff lemma also shows the converse that if fields have just a single branch then equilibria never underinvest in new fields under fixed-dispersion.

Proposition 3 If fixed dispersion holds then with positive probability an equilibrium will underinvest in new fields at some date if and only if $T \geq 4$ and the number of branches in fields is greater than or equal to 2.

As in the certain-continuation model, underinvestment becomes near certain to occur at some date when $T$ is large and the returns to project quality in a given field are diminishing. See Appendix A for formal definitions of the terminology in this proposition.

Proposition 4 Suppose fixed dispersion holds and that $\delta<1$. With probability 1 any equilibrium sequence underinvests in new fields at some date if and only if the number of branches in each field is greater than or equal to 2.

To sketch the argument for Proposition 4, notice that if $\delta<1$ the expected values that a field generates following an arbitrary $h_{t-1}$ will decrease geometrically through time. Consequently the sum of the expected values generated in a given field from $t$ through the end of the model $T$ will remain bounded above as $T$ increases and an existing field whose leading projects have low value will in expectation generate a low sum of values no matter how large $T$ is. But the fall-back advantage that comes from a new field's multiplicity of branches does not diminish as $T$ increases. Consequently there will be a cutoff $c$ that works for all values of $T$ : whenever project values fall below this $c$ new field initiation will deliver a social benefit. To conclude that an equilibrium will be practically sure to underinvest in new fields if the time span $T$ of the model is long enough, one need only argue that the number of new fields initiated in equilibrium keeps increasing as $T$ increases: since each time a new field is initiated its value will fall into the interval $(0, c)$ with positive probability, the probability that one new field will have such a value will converge to 1 as $T$ increases. The proof of the missing step (that the number of new fields initiated increases without bound) is an application of the martingale convergence theorem. 


\section{Secretive risk-takers make the best scientists}

\subsection{The superiority of risky projects}

Consider a choice between two projects $\pi$ and $\tilde{\pi}$ that have the same expected value but where one of the projects is riskier than the other. Such decisions arise when choosing a field to initiate or when choosing which branch of a root project to follow; or the decision could just be a comparative statics experiment that replaces one feasible project with another. Although both $\pi$ and $\tilde{\pi}$ in expectation generate the same value at the date the choice is made, the decision can affect the expected value achieved later on.

Let $t$ be the date the decision is made and let $\bar{\pi}$ be the best alternative among the projects available at $t$ but not chosen $(\bar{\pi}$ could be whichever project, $\pi$ or $\tilde{\pi}$, is not chosen at $t$ ). The riskier project is the superior choice for the curious reason that its greater dispersion means it is more likely to have a value that falls below that of the value of the best available alternative $\bar{\pi}$. Consequently when the successors of the riskier project are abandoned the gain from turning to $\bar{\pi}$ is greater on average than with the less risky project. This reasoning recalls our random walk illustration (section 3.1): scientists can achieve a stream of strictly positive expected values due to the opportunity of turning to new fields with 0 expected value whenever projects in existing fields turn negative.

Formally, given two feasible projects $\pi$ and $\tilde{\pi}$ with the same expected value, we consider $\tilde{\pi}$ to be riskier than $\pi$ if the distribution of $v(\pi)$ second-order stochastically dominates the distribution of $v(\tilde{\pi})$. Second-order stochastic dominance can be defined in several equivalent ways (Rothschild and Stiglitz (1970)); for our purposes it is easiest to apply mean-preserving spreads. We now fix some history; all probabilities and expectations should be read as conditional on that history. Given two feasible projects $\pi$ and $\tilde{\pi}$, we define $\tilde{\pi}$ to be riskier than $\pi$ if $v(\tilde{\pi})$ has the same distribution as the sum of $v(\pi)$ and a mean-preserving spread, that is, if there is a random variable $Z$ such that $(1) E(Z \mid v(\pi)=v)=0$ for all $v \in \mathbb{R}$, and (2) $P(v(\tilde{\pi}) \leq y)=P(v(\pi)+Z \leq y)$ for all $y \in \mathbb{R}^{8}{ }^{8}$

Suppose that in the period after $\pi$ or $\tilde{\pi}$ is chosen a successor of the selected project is undertaken if and only if its expected value is greater than the expected value $\bar{v}$ of the project

\footnotetext{
${ }^{8}$ To accommodate $Z$, let the state space now be $\Omega \times \mathbb{R}$.
} 
$\bar{\pi}$ designated as the best available alternative; otherwise $\bar{\pi}$ is chosen. We assume that $\bar{v}$ is unaffected by the choice between $\pi$ and $\tilde{\pi}$. Also we say that $\tilde{\pi}$ 's greater risk is nontrivial relative to $\bar{v}$ if there is a positive chance that $Z$ when added to $v(\pi)-\bar{v}$ can change its sign, that is, if $P((v(\pi)-\bar{v})(v(\pi)-\bar{v}+Z)<0)>0$. The Proposition below holds for any model, not just those introduced in section 4 .

Proposition 5 If following an arbitrary history $\tilde{\pi}$ is riskier than $\pi$ then the expected value of research in the next two periods will be at least as great if $\tilde{\pi}$ rather than $\pi$ is undertaken. If $\tilde{\pi}$ 's greater risk is nontrivial relative to the value of the best available alternative then the expected value achieved will be strictly higher with $\widetilde{\pi}$.

Over a time horizon longer than two periods, it is possible that a riskier project will lower the sum of expected values of research: consider for example a scenario where the riskier project $\tilde{\pi}$ has successors whose values are less risky conditional on $v(\widetilde{\pi})$. But if we embed a choice between more and less risky projects into the certain-continuation or fixed-dispersion models and assume that future project decisions are made optimally (they maximize the total expected value of research) then the riskier project is always the superior choice.

\subsection{Secrecy partially offsets the externality}

We have assumed so far that each scientist chooses at a single date. The assumption has not carried much significance since if a scientist chooses multiple projects but at dates that are far apart then the selection at earlier dates will have only a slight impact on the value of available choices later on (when $\delta<1$ ). But if a scientist chooses at multiple dates and can keep the results of his or her research secret - say by delaying publication - then the scientist can initiate a new field and, when field's root project has great value, reap the rewards of its high-value continuation projects. The externality under discussion is then alleviated.

For example, in the certain-continuation model a scientist who undertakes a single project will never initiate a new field as long as some existing field has projects with positive value. Suppose now that a scientist can conduct $\tau$ projects in secrecy and that other scientists will not know enough of the details of these projects to undertake any of their successor projects 
until the scientist finally releases his research. ${ }^{9}$ The scientist undertaking the secret research could then well prefer to initiate a new field. If $\bar{v}$ is the average value that the scientist can achieve by pursuing $\tau$ projects in existing fields then the scientist will be better off initiating a new field if $\sum_{j=1}^{\tau} \delta^{\left\lceil\frac{j}{\beta}\right\rceil} \int_{\bar{v}}^{\infty}(v-\bar{v}) g(v) d v>\bar{v}$, where we assume that $\delta \leq 1,\lceil x\rceil$ denotes the smallest integer at least as great as $x$, and recall that $\beta$ is the number of branches in a tree. $^{10}$

Similar results apply to the fixed-dispersion model. We can reverse the original purpose of the Cutoff Lemma to conclude that if $\beta \geq 2$ and $\tau \geq 3$ then a scientist who can keep secrets will be better off initiating a new field if the value of projects in existing fields is sufficiently small.

Of course a scientist working in secret will not internalize the whole of the externality considered in section 4. As long as $T$, the time span of the entire model, is greater than $\tau$, the number of projects a scientist can secretly undertake, scientists will continue to suboptimally ignore some of the socially beneficial consequences of new field initiation. But the present analysis does suggest a more generous view of scientists who cagily refuse to discuss their work; even if motivated by paranoia their secrecy could well foster the initiation of new lines of inquiry, which is a socially productive goal.

\section{Costs and benefits of free speech}

When scientists work in isolation, ignorant of what others have accomplished, then clearly it could be beneficial, both for the scientists and for society at large, for the scientists to learn the results of their peers' research: some scientists might be pursuing projects of little value while their peers are achieving great things. This section makes the reverse case, that communication can sometimes be welfare diminishing.

\footnotetext{
${ }^{9}$ Other scientists might of course repeat the entire sequence of projects that the scientist under consideration has undertaken in secret. But when the sequence begins with the initation of a new field our assumption of an ample supply of new fields means that this scenario is remote. Also, as we will see in section 6 , if other scientists know which projects are being undertaken in secret they will not want to replicate them.

${ }^{10}$ The inequality is a sufficient but not a necessary condition for the initiation of a new field to be an optimal action. Given that if $\delta<1$ and $(b, i, f)$ is the $j$ th project in $f$ undertaken after the root project then $i=\left\lceil\frac{j}{\beta}\right\rceil$, the inequality we have stated requires only a slight adaptation of the proof of Proposition 1 .
} 
We assume that, for any project $\pi$ and any history $h_{t}$ such that $\pi$ is not in $h_{t}$, the conditional distribution of $v(\pi)$ given $h_{t}$ admits a density.

We now divide the world of scientists into separate groups that we call 'countries.' The scientists in any one country are aware of which projects are undertaken abroad but either do not know the value they have delivered or do not know enough about the projects to undertake their successors. Since as before we assume that each scientist aims to maximize the additional value generated by his or her scientific research, scientists will not repeat the projects that have been already undertaken in other countries.

Let there be $n \geq 2$ countries who choose projects in a fixed sequence from periods 1 through $T$, where $T$ is a multiple of $n$. So country $k$ chooses at $t$ if there is an integer $j \geq 0$ such that $t=k+j n$. Also, given the history $h_{t-1}$, we define project $(b, i, f)$ to be feasible under autarchy at $t$ if $(b, i, f)$ is feasible as defined in section 2 and if, when $i \geq 1,(b, i-1, f)$ is chosen in history $h_{t-1}$ by the same country that chooses at $t$.

Definition 2 Given the history $h_{t-1}$, a project $\pi$ is an autarchy equilibrium choice if

- $\pi$ is feasible under autarchy at $t$,

- $E\left(v(\pi) \mid h_{t-1}\right) \geq E\left(v\left(\pi^{\prime}\right) \mid h_{t-1}\right)$ for all $\pi^{\prime}$ that are feasible under autarchy at $t$.

An autarchy equilibrium is a plan $\left(a_{1}, \ldots, a_{T}\right)$ such that each $a_{t}$ assigns an autarchy equilibrium choice to each history $h_{t-1}$.

Suppose that, beginning from autarchy, the barriers to communication fall at some date $t$ - say the internet appears at $t$ - giving each country full information about the projects undertaken by the other countries and allowing each country $k$ to choose any feasible project whenever it is $k$ 's turn to choose. Given the autarchy equilibrium $\left(a_{1}, \ldots, a_{T}\right)$, we say that full communication begins at $t$ to refer to a plan that consists of $a_{1}, \ldots, a_{t-1}$ but then, for $i=t, \ldots, T$, an $a_{i}^{\prime}$ that assigns an equilibrium choice (in the original sense of Definition 1) to each history $h_{i-1}$.

When full communication begins at $t$, the immediate response is for new field initiation to fall relative to what would happen if autarchy were to continue. This initial effect holds for any model, not just in the certain-continuation and fixed-dispersion special cases, and lasts 
for at least $n$ periods. To see what drives this decline, notice that if the autarchy equilibrium were to continue beyond $t$ then, with probability 1 , a new field would be initiated in one of the next $n$ periods, say $i$, if all of the projects feasible under autarchy for the country that chooses at $i$ have negative expected value. If, however, full-communication begins at $t$ then the country that chooses at $i$ will have the additional option of choosing projects from fields initiated by other countries and these projects may have positive expected value. So the chooser at $i$ under full-communication will initiate a new field only if every feasible project in every field previously initiated by some country has nonpositive expected value. Consequently, if there are $K$ countries that would choose from existing fields from $t$ through $t+n-1$ if autarchy were to continue then there must be at least $K$ projects that must be undertaken once full communication begins before any country will initiate. ${ }^{11}$

Proposition 6 In the first $n$ periods after full communication begins, the number of new fields initiated will with probability 1 be no greater than the number of new fields that would be initiated in the same $n$ periods if autarchy were to continue. With positive probability the number of new fields initiated will be strictly smaller in the first $n$ periods after full communication begins.

Proof. The remaining 'positive probability' claim follows, for example, from the fact that it is a positive probability event for each country prior to the beginning of full communication at $t$ to select only negative-value root projects under autarchy and for the country that chooses at $t$ (under autarchy and under full communication) to select a positive-value root project, say in field $f$. Under full communication the country that chooses at $t+1$ will then choose from $f$, while if autarchy were to continue every country from $t+1$ to $t+n-1$ would initiate.

In states where at $t$ the values of leading projects in existing fields are sufficiently low, a transition to full communication will suboptimally delay the initiation of new fields. For example, we have:

\footnotetext{
${ }^{11}$ The reasoning we have given applies with probability 1 and not to every state due to the knife-edge possibility that a country could decide under autarchy to undertake a project in a field that the country has already initiated even though that project has 0 expected value but, when full communication begins, instead decide to initiate a new field (assuming every feasible project has 0 expected value).
} 
Proposition 7 In the certain-continuation model, for any date $t$ with $n-1<t \leq T-n$ and any autarchy equilibrium there is a positive probability set $H$ of histories from 1 to $t-1$ such that, given any $h_{t-1} \in H$,

- the autarchy equilibrium initiates a new field at $t$,

- if full communication begins at $t$ then a new field will not be initiated at $t$,

- the conditional expected value of research from $t$ to $T$ given $h_{t-1}$ is greater in the autarchy equilibrium.

The Proposition is easily proved: it is a positive probability event that through date $t-1$ every country but the last country $n$ draws a root project with a positive value near 0 and that $n$ draws only negative value root projects. The expected value of research from $t$ to $T$ will be higher if $n$ draws again at the first date at $t$ or later at which it chooses, as it will under autarchy but will not under full communication.

\section{The citations game}

As we have seen, when scientists care only about the value of their own projects they ignore the riskiness and multiple branch advantages of new fields; the result is that too few new fields are initiated. It might seem that citations will fix at least the multi-branch externality since the initiator of a new field $f$ will then get a citation credit from the projects undertaken in every branch of $f$. But as we will see, since a new field must prove to be sufficiently valuable for it to earn citations, citation seekers can sometimes make the same decisions as the value-maximizing scientists we have considered so far.

Suppose that scientists cite all of the work that made their choice of project $\pi$ possible - all of the projects in the same field that are predecessors of $\pi$ - and that each scientist seeks to maximize the number of projects that cite his or her work. More precisely, agents $1, \ldots, T-1$ will maximize their expected number of citations and agent $T$ will maximize the expected value of research undertaken in period $T$. Nothing in our analysis would change if instead all agents were to maximize a weighted sum of their expected number of citations 
and the expected value of the research they personally undertake as long as the weight on citations is sufficiently large. We will now call the agents and equilibria of section 3 valueseeking. The pursuit of citations introduces a strategic dimension that did not appear in the value-seeking model; scientist $t$ 's choice of project will now be shaped by $t$ 's expectations of which projects future scientists will cite.

To see that citations need not cure the underinvestment externality even when fields have multiple branches, reconsider the certain continuation model when $\delta$ equals 1 - the simplest case since all projects in a field then share a common value. In a value-seeking equilibrium, once a field $f$ has been revealed to have positive-value projects every subsequent scientist piles into $f$. Citation maximizers can behave the same way. Suppose at some date $t$ that field $f$ has the highest-value projects with common value $\bar{v}>0$. If the scientist at $t$ initiates a new field $f^{\prime}$ then $t^{\prime}$ 's expected number of citations can be no larger than $(T-t) P\left(v\left(0, f^{\prime}\right) \geq \bar{v}\right)$, the number of remaining agents multiplied by the probability that $f^{\prime}$ generates projects with value greater than $\bar{v}$ (if $v\left(0, f^{\prime}\right)<\bar{v}$ then scientist $T$ will refuse to choose a project in $f^{\prime}$ and by backward induction every other scientist will also refuse). If on the other hand $t$ undertakes a project in $f$ and subsequent agents do the same and, to avoid artificial asymmetries, we assume that each agent randomizes by placing equal weight on each of the $\beta$ available projects in $f$, then $t$ will earn $(T-t) \frac{1}{\beta}$ expected citations. Thus if

$$
P\left(v\left(0, f^{\prime}\right) \geq \bar{v}\right) \leq \frac{1}{\beta}
$$

then it will be an equilibrium for every agent from $t$ onwards to pursue a project in $f$ rather than initiate. Each of these agents will be happy to undertake a project in $f$ given that every later agent does the same. The converse holds as well: with our assumed randomization behavior, if (7.1) is violated then in any equilibrium some agent will initiate a new field. ${ }^{12}$

Now if we assume as a benchmark case that the density $g$ that governs $v\left(0, f^{\prime}\right)$ is symmetric around 0 then $P\left(v\left(0, f^{\prime}\right) \geq \bar{v}\right)$ must be smaller than $\frac{1}{2}$ and hence (7.1) will in fact be satisfied when $\beta \leq 2$. So under these conditions citation maximizers will exactly reproduce the behavior of value seekers: agents will initiate new fields until they come to the first field

\footnotetext{
${ }^{12}$ Agents $t, \ldots, T$ cannot all decline to initiate since then agent $T-1$ would deviate: he earns one citation with probability $P\left(v\left(0, f^{\prime}\right) \geq \bar{v}\right)$ if he initiates and $\frac{1}{\beta}$ expected citations if he undertakes a project in $f$.
} 
with a positive value and then all subsequent agents pile in. Moreover, for larger values of $\bar{v}$, (7.1) will be satisfied for larger values of $\beta$. So, for any number of branches, if a field has been discovered with sufficiently high value all future new field initiation will come to an end. But new field initiation may well continue to be optimal. Since $T-t$ scales the magnitude of the social gain when a new field surpasses the value of existing fields in the $\delta=1$ model (see (4.1) in section 4.1), for any $\bar{v}$ less than the maximum conceivable value further new field initiation will be optimal when the number of remaining periods $T-t$ is sufficiently large. In contrast the number of periods remaining has no bearing on equilibrium condition (7.1). The failure of citation maximization to generate sufficient investment in new fields is thus endemic in the certain continuation model.

Our analysis has stepped around some potential equilibrium oddities by assuming that, when multiple projects in a single field $f$ lead to the same expected number of citations or have the same expected value, agents select each of these projects with equal probability. We now show how to handle randomized decisions and how to cover models where $\delta \neq 1$. For simplicity only, we assume that agents do not mix when they initiate a new field.

Previously the function $a_{t}$ specified a single feasible action for each $h_{t-1}$, namely the project that $t$ selects following $h_{t-1}$. Now $a_{t}\left(h_{t-1}\right)$ will denote a set of feasible projects for each $h_{t-1}$, the projects that $t$ chooses with positive probability. In addition to specifying values for all projects, a state $\omega$ will now also indicate the outcome of each agent's randomization, i.e., the particular project in $a_{t}\left(h_{t-1}\right)$ that $t$ actually selects. ${ }^{13}$ We assume that agents mix with equal probability across the projects they select. ${ }^{14}$ A history $h_{t}$ will now in addition specify the outcomes of any randomizations that agents execute at $t$ or earlier.

Given the plan $a=\left(a_{1}, \ldots, a_{T}\right)$, agent $t$ earns the citation $\pi^{\prime}$ at state $\omega$ if (1) $\pi^{\prime}$ is a direct or indirect successor of the project $\pi$ that agent $t$ selects at $\omega$ and (2) some agent that chooses after $t$ selects $\pi^{\prime}$ at $\omega .{ }^{15} \quad$ A plan $a$ thus defines a total number of citations $C_{t}[a](\omega)$ for $t$ at $\omega$ and hence an expected number of citations $E\left(C_{t}[a]\right)=\int C_{t}[a](\omega) d P(\omega)$. In the

\footnotetext{
${ }^{13}$ Formally, a state $\omega$ specifies, for every plan $a$ and history $h_{t-1}$, a project in $a_{t}\left(h_{t-1}\right)$, which is to be interpreted as the project that $t$ 's randomization selects given $a$ and $h_{t-1}$. The definition of the set of states at which $\pi$ is undertaken at date $t,[\pi]_{t}$, proceeds comparably to Appendix A.

${ }^{14}$ Formally, we suppose that, for all $a$ and all $h_{t-1}$, if $\pi, \pi^{\prime} \in a_{t}\left(h_{t-1}\right)$ then (using the notation given in Appendix A) $P\left([\pi]_{t} \mid h_{t-1}, a\right)=P\left(\left[\pi^{\prime}\right]_{t} \mid h_{t-1}, a\right)$.

${ }^{15}$ These requirements can be stated as $(1) \omega \in[\pi]_{t}$ and $(2) \omega \in\left[\pi^{\prime}\right]_{i}$ for some $i>t$.
} 
definition below, we use $a^{\left\langle\pi, h_{t-1}\right\rangle}$ to denote the plan that coincides with $a$ in every coordinate except that $a_{t}^{\left\langle\pi, h_{t-1}\right\rangle}\left(h_{t-1}\right)=\{\pi\}$.

Definition 3 A citations equilibrium is a plan $a=\left(a_{1}, \ldots, a_{T}\right)$ such that

- for each $t=1, \ldots, T-1$ and $h_{t-1}$, there is a field $f$ such that $(b, i, f) \in a_{t}\left(h_{t-1}\right)$ if and only if $(b, i, f)$ is feasible following $h_{t-1}$ and $E\left(C_{t}\left[a^{\left\langle(b, i, f), h_{t-1}\right\rangle}\right] \mid h_{t-1}\right) \geq E\left(C_{t}\left[a^{\left\langle\pi^{\prime}, h_{t-1}\right\rangle}\right] \mid h_{t-1}\right)$ for all $\pi^{\prime}$ that are feasible following $h_{t-1}$,

- for each $h_{T-1}$, there is a field $f$ such that $(b, i, f) \in a_{T}\left(h_{T-1}\right)$ if and only if $E\left(v(b, i, f) \mid h_{T-1}\right)$ $\geq E\left(v\left(\pi^{\prime}\right) \mid h_{T-1}\right)$ for all $\pi^{\prime}$ that are feasible following $h_{T-1}$.

By viewing the $a_{t}$ functions as strategies, a citations equilibrium qualifies as a Nash equilibrium; that an equilibrium assigns an optimizing choice to each history amounts to a subgame perfection requirement.

As we have seen, if in the certain continuation model $\delta=1$ and $\beta \leq 2$ and given a mild restriction on the distribution of project values a citations equilibrium will replicate the behavior of a value-seeking equilibrium. The next Proposition shows that if $\delta>1$ we can drop the restriction on likelihoods and if $\delta<1$ then the replication conclusion will hold in (loosely speaking) half of the possible cases. Value-seeking behavior thus predominates when $\beta$ is no larger than 2 and we conclude that a move to citation-maximization need not increase welfare, even when branches are multiple.

Given a state $\omega$, a citations equilibrium determines a path of $T$ actions. Since a state defines values for all projects, we can check whether there is a value-seeking equilibrium that leads to the same equilibrium path of actions when the same project values obtain. If so, we say that 'the path of actions taken can be taken in a value-seeking equilibrium.'

Proposition 8 In the certain-continuation model,

- if $\delta>1$ then, for any citations equilibrium, with probability 1 the path of actions taken can also be taken in a value-seeking equilibrium,

- if $\delta=1$ and (i) the probability that a root project has positive value is no greater than $\frac{1}{2}$ and (ii) $\beta \leq 2$, then, for any citations equilibrium, with probability 1 the path of actions taken can also be taken in a value-seeking equilibrium, 
- if $\delta<1$ and (i) and (ii) hold, then for any citations equilibrium the path of actions taken can also be taken in a value-seeking equilibrium if the state is such that an odd number of periods remain after the first project with a positive value is revealed.

So, in the $\delta>1$ case, the citations equilibria will exactly track the behavior of the valueseeking model and reproduce its underinvestment externalities. While the same conclusions hold in the $\delta=1$ case only under restrictions, we saw earlier that this model is sure to display an underinvestment externality when the time span $T$ is long enough.

The $\delta<1$ case is inherently more complicated. The qualifications needed in this case stem from a curious end-of-model effect. For example, when $\beta=2$, the date is $T-1$, and the same number of projects have been undertaken in each branch of the only field $f$ revealed to have positive-value projects, the scientist at $T-1$ will not undertake a project in $f$ (as he would in a value-seeking equilibrium) since scientist $T$ will then choose from the branch of $f$ that $T-1$ did not choose from. This extra new field initiation at $T-1$ then unravels the potential for a citations equilibrium to mimic value-seeking actions at earlier dates.

While we have so far emphasized that citation maximization can lead to underinvestment in new fields, it can also lead to overinitiation of new fields. The easiest way to see this possibility is again in the certain continuation model with $\delta=1$. Let the highest value among projects available at some date $t$ be denoted as usual by $\bar{v}$ and assume that any further initiation of new fields is suboptimal, i.e., following (4.1) suppose that $(T-t) \int_{\bar{v}}^{\infty}(v-$ $\bar{v}) g(v) d v<\bar{v}$. Since this condition does not restrict the number of branches $\beta$, new field initiation can be suboptimal even when $\beta$ is large enough to ensure that (7.1) is violated and hence that new fields will be initiated in any citations equilibrium.

Notice the parallelism with our earlier discussion: for given $\beta$ we saw that if the remaining number of periods $T-t$ is large enough then new field initiation is optimal but will not occur in a citations equilibrium, while here we have seen that for given $T-t$ if $\beta$ is large enough then new field initiation is suboptimal but will occur in citations equilibrium.

If we step outside of the confines of the model and let fields have a variety of different $\beta$ 's, $\delta$ 's, and expected values, the inefficiency of citation maximization becomes transparent: citation maximizers will avoid fields whose root projects have enormous expected value but tiny $\delta$ 's in favor of fields with lower initial expected value but high $\delta$ 's. 
We have supposed that the results of any project will spread to the entire scientific community. But if journals oversee the dissemination of research and thus the flow of citations then scientists will take on only those projects that journals will publish. Unfortunately the root project of a new field will not cite any past work and hence no citation-seeking editor or referee will have an incentive to let the research pass through the gate. The path by which citations potentially can mitigate underinvestment in new fields may therefore be blocked. There are countervailing forces: editors may derive a citation-like credit from stewarding a journal that initiates new fields. For anonymous citation-seeking referees, however, there is no cost to snuffing out a new field.

\section{Conclusion}

The individual pursuit of scientific value - or the pursuit of the rewards that accompany successful scientific careers - does not necessarily maximize the total value produced by the entire community of scientists. Even when scientists seek the recognition of other researchers in the form of citations, they may avoid innovative projects that could make a rich supply of spin-offs available. On the other hand, a taste for risk and secrecy - not traits that academia normally encourages - can ease the externality.

Our analysis has been geared to scientific research but it applies to any pursuit where individual projects or works can be ranked as better or worse, and where projects in a specific area build on the work done earlier. We mentioned the technological interpretation of the model in section 2. The trees we have used to link a scientific project to earlier work could also describe the bridge between past and present in cultural and artistic endeavors. And individuals in these fields can also pursue a citations-like credit for the work they stimulate.

\section{Appendix A: technical definitions}

To define the probability that a specific project is undertaken in a given period, let $v(\pi, \omega)$ denote the value realized for project $\pi$ in state $\omega$, let $h_{0}$ denote the null history that agents face in period 1 , and let $\Omega$ denote the entire set of states. Given plan $a=\left(a_{1}, \ldots, a_{T}\right)$, the set of states where project $\pi$ is undertaken at date $t$, which we write as $[\pi]_{t}$, and the set of 
states where $\pi$ is undertaken at date $t$ and realizes value $v$, written $[\pi, v]_{t}$, are defined by

$$
\begin{aligned}
& {[\pi]_{1}=}\left\{\omega: a_{1}\left(h_{0}\right)=\pi\right\} \text { (equal to either } \varnothing \text { or } \Omega \text { ) } \\
& {[\pi, v]_{1}=}\left\{\omega: a_{1}\left(h_{0}\right)=\pi \text { and } v(\pi, \omega)=v\right\} \\
& {[\pi]_{2}=}\left\{\omega: \exists v_{1}, \pi_{1} \text { such that } a_{1}\left(h_{0}\right)=\pi_{1}, v\left(\pi_{1}, \omega\right)=v_{1}, a_{2}\left(\pi_{1} ; v_{1}\right)=\pi\right\} \\
& {[\pi, v]_{2}=}\left.\left\{\omega: \exists v_{1}, \pi_{1} \text { such that } a_{1}\left(h_{0}\right)=\pi_{1}, v\left(\pi_{1}, \omega\right)=v_{1}, a_{2}\left(\pi_{1} ; v_{1}\right)\right)=\pi, v\left(\pi_{2}, \omega\right)=v\right\} \\
& \vdots \\
& {[\pi]_{t}=}\left\{\omega: \exists\left(v_{i}, \pi_{i}\right)_{i=1}^{t-1} \text { such that } a_{i}\left(\left(\pi_{j}\right)_{j=1}^{i-1} ;\left(v\left(\pi_{j}, \omega\right)\right)_{j=1}^{i-1}\right)=\pi_{i} \text { and } v\left(\pi_{i}, \omega\right)=v_{i}\right. \\
&\left.\quad \text { for } i=1, \ldots, t-1 \text { and } a_{i}\left(\left(\pi_{j}\right)_{j=1}^{t-1} ;\left(v\left(\pi_{j}, \omega\right)\right)_{j=1}^{t-1}\right)=\pi\right\} \\
& {[\pi, v]_{t}=}\left\{\omega: \exists\left(v_{i}, \pi_{i}\right)_{i=1}^{t-1} \text { such that } a_{i}\left(\left(\pi_{j}\right)_{j=1}^{i-1} ;\left(v\left(\pi_{j}, \omega\right)\right)_{j=1}^{i-1}\right)=\pi_{i}, v\left(\pi_{i}, \omega\right)=v_{i}\right. \text { for } \\
&\left.i=1, \ldots, t-1, \text { and } a_{i}\left(\left(\pi_{j}\right)_{j=1}^{t-1} ;\left(v\left(\pi_{j}, \omega\right)\right)_{j=1}^{t-1}\right)=\pi, v(\pi, \omega)=v\right\} .
\end{aligned}
$$

Although our notation will not indicate the dependence, keep in mind that the events $[\pi]_{t}$ and $[\pi, v]_{t}$ are always defined relative to a plan $a$.

Probabilities are defined as usual from the relevant sets of states; for example the probability that project $\pi$ is undertaken at date $t$ when plan $\left(a_{1}, \ldots, a_{T}\right)$ is adopted is $P\left([\pi]_{t}\right)$. We define total expected value of research achieved by a to be

$$
\int_{\Omega} \sum_{[\pi, v]_{i}: \omega \in[\pi, v]_{i}} v d P(\omega) .
$$

To remove any trace of ambiguity: given a $\omega \in \Omega$ the summation above is taken over all $[\pi, v]_{i}$ such that $\omega \in[\pi, v]_{i}$ for some $i \in\{1, \ldots, T\}$, project $\pi$, and $v \in \mathbb{R}$. Since $\omega$ specifies a value for each project and there are only finitely many projects that can be undertaken by period $T$, there are only finitely many such $[\pi, v]_{i}$ and hence the summation is well-defined.

We can also define the event where the history $h_{t}=\left(\pi_{1}, \ldots, \pi_{t} ; v\left(\pi_{1}\right), \ldots, v\left(\pi_{t}\right)\right)$ occurs given $a$ by $\left[h_{t}\right]=\bigcap_{i=1, \ldots, t}\left[\pi_{i}, v\left(\pi_{i}\right)\right]_{i}$, and accordingly the probability of a set of histories. Given $a$, we say that a state $\omega \in \Omega$ leads to the history $h_{t}$ if $\omega \in\left[h_{t}\right]$.

We define an equilibrium a to underinvest in new fields following $h_{t-1}$ if there exists an alternative plan $a^{\prime}$ such that, for all $\omega$ that lead to $h_{t-1}, a_{t}$ selects a successor of a leading project following $h_{t-1}$ while $a^{\prime}(1)$ is identical to $a$ up to $t-1$, (2) initiates a new field following $h_{t-1}$, and (3) increases the conditional total expected value of research given $\omega$.

When the particular $t$ at which underinvestment occurs is immaterial, we say that an equilibrium a underinvests in new fields at some date given $\omega$ if there is an alternative plan $a^{\prime}$ such that (i) for all $t$ and all $h_{t-1}$, if $a$ initiates a new field following $h_{t-1}$ then so does $a^{\prime}$ and (ii) $a^{\prime}$ achieves a greater conditional total expected value of research than $a$ given $\omega .{ }^{16}$ Details aside, the primary way $a^{\prime}$ can do better in expectation than $a$ is to initiate new fields at histories where $a$ does not.

To consider the consequences of letting the time horizon of the model increase, define $\left(a_{1}, \ldots, a_{t}, \ldots\right)$ to be an equilibrium sequence if, for $\tau \geq 1$, the plan of length $\tau,\left(a_{1}, \ldots, a_{\tau}\right)$,

\footnotetext{
${ }^{16}$ At the cost of lengthening some of the proofs, we could additionally require that $a$ and $a^{\prime}$ are identical except that at some histories $a^{\prime}$ initiates a new field while $a$ does not.
} 
forms an equilibrium (for $T=\tau$ ). We will say that with probability 1 a sequence underinvests in new fields at some date if there is a set of states $A$ with $P(A)=1$ where, for any $\omega \in A$, there is a $L$ such that any plan $\left(a_{1}, \ldots, a_{\tau}\right)$ in the sequence with $\tau \geq L$ underinvests in new fields at some date given $\omega$.

\section{Appendix B: remaining proofs}

Proof of Proposition 2. The only detail omitted from the text in the $\delta=1$ case is that it is a probability 0 event for the first $(0, f)$ with $v(0, f)>0$ undertaken by the equilibrium sequence to have $v(0, f)=v_{\max }$, where $v_{\max }=\sup \{v: g(v)>0\}$. Hence, with probability $1, v(0, f)$ lies in $\left(0, v_{\max }\right)$. It follows that, with probability $1,(4.1)$ is satisfied when $T$ is sufficiently large. Next, assume that $\delta<1$. Given that the distribution of the value of root projects admits a density, with probability 1 an equilibrium sequence will initiate a field $f$ at some $t$ whose root project has value $\bar{v}>0$. The expected value of research achieved through any date $\tau$ is therefore bounded above by $\lim _{n \rightarrow \infty} \sum_{i=0}^{n} \delta^{i} \beta \bar{v}$. As an alternative, let $v^{\prime}>0$ be an arbitrary element of the interior of the support of $g$ and consider the sequence that initiates fields until some $f_{1}$ has $v\left(0, f_{1}\right) \geq v^{\prime}$, then undertakes $\left(1,1, f_{1}\right)$, and then returns to initiating new fields until some $f_{2}$ has $v\left(0, f_{2}\right) \geq v^{\prime}$, and so on. The Doob stopping theorem (see, e.g., Williams (1991)) implies that the conditional expected value achieved from date 1 through $t_{1}$, given that $\left(0, f_{1}\right)$ is undertaken at $t_{1}$, equals 0 and hence the conditional expected value achieved from 1 through $t_{1}+1$, given that $\left(1,1, f_{1}\right)$ is undertaken at $t_{1}+1$, is bounded below by $\delta v^{\prime}$. Similarly, for any $i \geq 1$, the conditional expected value achieved from $t_{i}+2$ through $t_{i+1}+1$, given that $\left(1,1, f_{i}\right)$ is undertaken at $t_{i}+1$ and $\left(1,1, f_{i+1}\right)$ is undertaken at $t_{i+1}+1$, is also bounded below by $\delta v^{\prime}$. Since the event where infinitely many initiations in a row all reveal root projects with value less than $v^{\prime}$ has probability 0 , it follows that for any constant $k$ (including $\lim _{n \rightarrow \infty} \sum_{i=0}^{n} \delta^{i} \beta \bar{v}$ ) there is a $T$ by which this alternative strategy achieves total expected value greater than $k$.

Proof of cutoff lemma. I. Suppose $\beta \geq 2$ and $t \leq T-2$. To calculate the expected value of research achieved by the equilibrium plan $a$ from $t$ onwards, we first calculate the expected values of research in periods $t$ through $t+2$.

Let $V_{t+2}\left(v_{-1}\right)$ be the expected value of research at date $t+2$ in equilibrium given a history $h_{t+1}$ where $v_{-1}$ is the maximum of 0 and the value of the highest-value leading project of $h_{t+1}$. Let $V_{t+1}\left(v_{-1}^{l}, v_{-1}^{h}\right)$ be the sum of the expected value of research at dates $t+1$ and $t+2$ in equilibrium given a $h_{t}$ where $v_{-1}^{h}$ (resp. $v_{-1}^{l}$ ) is the maximum of the value of the highest value (resp. second-highest value) leading project of $h_{t}$ and 0 , and let $V_{t}\left(v_{-1}^{l}, v_{-1}^{m}, v_{-1}^{h}\right)$ be the sum of the expected value of research from $t$ through $t+2$ in equilibrium given a $h_{t-1}$ where $v_{-1}^{h}\left(\right.$ resp. $\left.v_{-1}^{m}, v_{-1}^{l}\right)$ is the maximum of the value of the highest value (resp. second-highest 
value, third-highest value) leading project of $h_{t-1}$ and 0 . We have

$$
\begin{aligned}
V_{t+2}\left(v_{-1}\right)= & \delta v_{-1}, \\
V_{t+1}\left(v_{-1}^{l}, v_{-1}^{h}\right)= & \delta v_{-1}^{h}+\int_{v_{-1}^{l}}^{\infty} V_{t+2}(v) g_{v_{-1}^{h}}(v) d v+\int_{-\infty}^{v_{-1}^{l}} V_{t+2}\left(v_{-1}^{l}\right) g_{v_{-1}^{h}}(v) d v \\
V_{t}\left(v_{-1}^{l}, v_{-1}^{m}, v_{-1}^{h}\right)= & \delta v_{-1}^{h}+\int_{v_{-1}^{m}}^{\infty} V_{t+1}\left(v_{-1}^{m}, v\right) g_{v_{-1}^{h}}(v) d v \\
& +\int_{v_{-1}^{l}}^{v_{-1}^{m}} V_{t+1}\left(v, v_{-1}^{m}\right) g_{v_{-1}^{h}}(v) d v+\int_{-\infty}^{v_{-1}^{l}} V_{t+1}\left(v_{-1}^{l}, v_{-1}^{m}\right) g_{v_{-1}^{h}}(v) d v .
\end{aligned}
$$

These formulas are mostly self-explanatory. The first term in the expression for each $V_{\tau}$ is the expected value of an immediate successor of the highest-value leading project of $h_{\tau-1}$, which will be project undertaken at $\tau$, while the remaining terms are the expectation of $V_{\tau+1}$ using the realization of the project undertaken at $\tau$ to determine the highest-value leading project of $h_{\tau+1}$. In the expression for $V_{t+1}\left(v_{-1}^{l}, v_{-1}^{h}\right)$, for example, the second integral indicates the fact that if the successor of the project with value $v_{-1}^{h}$ turns out to have value less than $v_{-1}^{l}$ then at date $t+2$ a successor of the project with value $v_{-1}^{l}$ will be selected.

Next consider an alternative plan that (i) at $t$, initiates a new field $f^{\prime}$, (ii) at $t+1$, selects a successor of $\left(0, f^{\prime}\right)$ if $v\left(0, f^{\prime}\right)>0$ and otherwise initiates a new field $f^{\prime \prime}$, and (iii) at $t+2$, selects a successor of $\left(0, f^{\prime}\right)$ or $\left(0, f^{\prime \prime}\right)$ if either $v\left(0, f^{\prime}\right)>0$ or $v\left(0, f^{\prime \prime}\right)>0$ and otherwise initiates a new field. Notice that with this strategy the expected values of research at $t$, $t+1$, and $t+2$ are not functions of the values of the leading projects of $h_{t}$ and the expected values at $t+1$ and $t+2$ are functions only of $v\left(0, f^{\prime}\right)$. So, letting $\widehat{V}_{\tau}$ denote the sum of the expected values of research from $t$ through $t+2$ for this alternative strategy and, at both $t+2$ and $t+1$, letting $v_{-1}$ denote the maximum expected value of leading projects of $h_{\tau}$ in fields $f^{\prime}$ or $f^{\prime \prime}$, we have

$$
\begin{aligned}
\widehat{V}_{t+2}\left(v_{-1}\right) & =\delta v_{-1}, \\
\widehat{V}_{t+1}\left(v_{-1}\right) & =\delta v_{-1}+\int_{v_{-1}}^{\infty} \widehat{V}_{t+2}(v) g_{v_{-1}}(v) d v+\int_{-\infty}^{v_{-1}} \widehat{V}_{t+2}\left(v_{-1}\right) g_{v_{-1}}(v) d v, \\
\widehat{V}_{t} & =\int_{0}^{\infty} \widehat{V}_{t+1}(v) g(v) d v+\int_{-\infty}^{0} \widehat{V}_{t+1}(0) g(v) d v .
\end{aligned}
$$

The key item above is the second integrand in the expression for $\widehat{V}_{t+1}\left(v_{-1}\right)$, which indicates that if the root project in the field initiated at $t$ has value $v_{-1}$ and the successor project undertaken at $t+1$ turns out to have value less than $v_{-1}$ then at $t+2$ a project in a different branch of the new field will be undertaken with an expected value of $\widehat{V}_{t+2}\left(v_{-1}\right)$.

We now compare the sum of expected values of research from $t$ through $t+2$ for these two strategies $-V_{t}\left(v_{-1}^{l}, v_{-1}^{m}, v_{-1}^{h}\right)$ versus $\widehat{V}_{t}-$ given various candidate cutoffs that we label as $\varepsilon(i)$. Let $\langle\varepsilon(n)\rangle$ be a sequence of strictly positive numbers such that $\varepsilon(n) \rightarrow 0$ and let $\left\langle v_{-1}^{l}(n), v_{-1}^{m}(n), v_{-1}^{h}(n)\right\rangle$ be a sequence of triples such that, for all $n, 0 \leq v_{-1}^{k} \leq \varepsilon(n)$ for $k=l, m, h$ and $v_{-1}^{l}(n) \leq v_{-1}^{m}(n) \leq v_{-1}^{h}(n)$, indicating the three highest values of leading projects at $t$, except that as before, since negative expected-value projects are not undertaken, 
0's replace negative values. Then $\delta v_{-1}^{h}(n) \rightarrow 0$ and $\int_{v_{-1}^{l}(n)}^{v_{-1}^{m}(n)} V_{t+1}\left(v, v_{-1}^{m}(n)\right) g_{v_{-1}^{h}(n)}(v) d v \rightarrow 0$ as $n \rightarrow \infty$. Substituting in the definitions of $V_{t+1}\left(v_{-1}^{l}, v_{-1}^{h}\right), V_{t+2}\left(v_{-1}\right), \widehat{V}_{t+1}\left(v_{-1}\right), \widehat{V}_{t+2}\left(v_{-1}\right)$, it is readily confirmed that

$$
\int_{-\infty}^{v_{-1}^{l}(n)} V_{t+1}\left(v_{-1}^{l}(n), v_{-1}^{m}(n)\right) g_{v_{-1}^{h}(n)}(v) d v \rightarrow \int_{-\infty}^{0} \widehat{V}_{t+1}(0) g(v) d v .
$$

Consider finally the remaining term in $V_{t}\left(v_{-1}^{l}(n), v_{-1}^{m}(n), v_{-1}^{h}(n)\right)$,

$$
\begin{aligned}
\int_{v_{-1}^{m}(n)}^{\infty} V_{t+1}\left(v_{-1}^{m}(n), v\right) g_{v_{-1}^{h}(n)}(v) d v= & \int_{v_{-1}^{m}(n)}^{\infty}\left[\delta v+\int_{v_{-1}^{m}(n)}^{\infty} \delta \widetilde{v} g_{v}(\widetilde{v}) d \widetilde{v}\right. \\
& \left.+\int_{-\infty}^{v_{-1}^{m}(n)} \delta v_{-1}^{m}(n) g_{v}(\widetilde{v}) d \widetilde{v}\right] g_{v_{-1}^{h}(n)}(v) d v
\end{aligned}
$$

and compare it to the remaining term in $\widehat{V}_{t}$,

$$
\begin{aligned}
\int_{0}^{\infty} \widehat{V}_{t+1}(v) g(v) d v & =\int_{0}^{\infty}\left[\delta v+\int_{v}^{\infty} \delta \widetilde{v} g_{v}(\widetilde{v}) d \widetilde{v}+\int_{-\infty}^{v} \delta v g_{v}(\widetilde{v}) d \widetilde{v}\right] g(v) d v \\
& =\int_{0}^{\infty}\left[\delta v+\int_{v}^{\infty} \delta \widetilde{v} g_{v}(\widetilde{v}) d \widetilde{v}+\int_{0}^{v} \delta v g_{v}(\widetilde{v}) d \widetilde{v}+\int_{-\infty}^{0} \delta v g_{v}(\widetilde{v}) d \widetilde{v}\right] g(v) d v
\end{aligned}
$$

Now, for any $v \geq 0$ and any $v_{-1}^{m}(n) \geq 0, \int_{v}^{\infty} \delta \widetilde{v} g_{v}(\widetilde{v}) d \widetilde{v}+\int_{0}^{v} \delta v g_{v}(\widetilde{v}) d \widetilde{v}>\int_{v_{-1}^{m}(n)}^{\infty} \delta \widetilde{v} g_{v}(\widetilde{v}) d \widetilde{v}$ which implies

$\lim _{n \rightarrow \infty} \int_{0}^{\infty}\left[\int_{v}^{\infty} \delta \widetilde{v} g_{v}(\widetilde{v}) d \widetilde{v}+\int_{0}^{v} \delta v g_{v}(\widetilde{v}) d \widetilde{v}\right] g(v) d v \geq \lim _{n \rightarrow \infty} \int_{v_{-1}^{m}(n)}^{\infty}\left[\int_{v_{-1}^{m}(n)}^{\infty} \delta \widetilde{v} g_{v}(\widetilde{v}) d \widetilde{v}\right] g_{v_{-1}^{h}(n)}(v) d v$

Since in addition

$$
\begin{aligned}
\int_{v_{-1}^{m}(n)}^{\infty} \delta v g_{v_{-1}^{h}(n)}(v) d v & \rightarrow \int_{0}^{\infty} \delta v g(v) d v, \text { and } \\
\int_{v_{-1}^{m}(n)}^{\infty}\left[\int_{-\infty}^{v_{-1}^{m}(n)} \delta v_{-1}^{m}(n) g_{v}(\widetilde{v}) d \widetilde{v}\right] g_{v_{-1}^{h}(n)}(v) d v & \rightarrow 0,
\end{aligned}
$$

the difference between (2) and (1) converges to a number at least as great as $\int_{0}^{\infty}\left[\int_{-\infty}^{0} \delta v g_{v}(\widetilde{v}) d \widetilde{v}\right] g(v) d v$, a strictly positive constant. Hence for all $n$ sufficiently large and hence all $\varepsilon(n)$ sufficiently small $\widehat{V}_{t}>V_{t}\left(v_{-1}^{l}(n), v_{-1}^{m}(n), v_{-1}^{h}(n)\right)$, which shows that for all sufficiently large $n$ the alternative strategy delivers larger expected value of research from $t$ to $t+2$ when $h_{t-1}$ is such that the highest value leading project has a value in $(0, \varepsilon(n))$.

We fill in the remainder of the alternative plan by defining project choices for periods $t+3, \ldots, T$ that will, as $\varepsilon(n) \rightarrow 0$, yield an expected value of research in these periods that converges to the expected value achieved by the equilibrium plan $a$ in the same periods. 
For $k=1, \ldots, T$, let $z_{k}$ denote a realization of the deviation of $v(\pi)$ from its expected value where $\pi$ is the project undertaken in equilibrium at $k$ when the deviations $z_{1}, \ldots, z_{k-1}$ have been realized. Given a history $h_{t+2}$, which is uniquely defined by the equilibrium plan $a$ and the realizations $z_{1}, \ldots, z_{t+2}$, suppose the equilibrium has $r$ projects feasible at $t+3$ with strictly positive expected value. Each of these projects must be in a distinct branch, which we label $1, \ldots, r$, of a single field $\widehat{f}$. It will be convenient to henceforth label the projects in these branches so that $(j, i, \widehat{f})$ for $i \geq 0$ now denotes the $(i+1)$ th successor in branch $j$ of the leading project at $t+3$ of the $j$ th branch of $\widehat{f}^{17}$ Let $f_{1}, \ldots$, $f_{r}$ index $r$ fields that the equilibrium plan $a$ has not initiated by date $t+2$. We may then define a 'preliminary' alternative plan $\alpha^{\prime}$ that undertakes project $\left(1, i, f_{j}\right)$ at period $t^{\prime}$, given that the projects undertaken earlier by $\alpha^{\prime}$ have realized deviations $z_{1}, \ldots, z_{t^{\prime}-1}$, whenever the equilibrium $a$, given the same deviations $z_{1}, \ldots, z_{t^{\prime}-1}$, undertakes $(j, i, \widehat{f})$. Finally, let $\alpha^{\prime}$, given the realized deviations $z_{1}, \ldots, z_{t^{\prime}-1}$, initiate a new field $f_{\text {alt }}$ (where $f_{\text {alt }} \notin\left\{f_{1}, \ldots, f_{r}\right\}$ ) whenever the equilibrium following the same deviations initiates a new field $f_{e q}$, and then, for $t^{\prime \prime}>t^{\prime}$, given the deviations $z_{1}, \ldots, z_{t^{\prime \prime}-1}$ undertake $\left(b, i, f_{\text {alt }}\right)$ whenever the equilibrium given the same deviations undertakes $\left(b, i, f_{e q}\right)$. Define a project undertaken at date $i$ to have a 'hypothetical value' equal to $\delta v+z_{i}$ when its predecessor has value $v$, even when $v<0$. We will now see that, given any $z=\left(z_{1}, \ldots, z_{T}\right)$, the sum of hypothetical values that $\alpha^{\prime}$ generates from $t+3$ to $T$ converges, as $\varepsilon(n) \rightarrow 0$, to the expected value generated by the equilibrium from $t+3$ to $T$. It follows (see part II below) that the plan $\alpha$ that is identical to $\alpha^{\prime}$ except that $\alpha$ initiates a new field whenever $\alpha^{\prime}$ undertakes a nonpositive expected value project must then both generate greater total expected value than the equilibrium $a$.

To conclude part I, therefore, we show that as $\varepsilon(n) \rightarrow 0$ the expected value of research from $t+3$ through $T$ under the equilibrium $a$ converges to the sum of the hypothetical expected values for the same periods under $\alpha^{\prime}$. Since fixed dispersion implies that each measurable set of deviation vectors $z=\left(z_{1}, \ldots, z_{T}\right)$ has the same probability in the two strategies, it is sufficient to show that, for any $z$, the difference between the expected value of research from $t+3$ to $T$ delivered by $a$ and the hypothetical values delivered by $\alpha^{\prime}$ in the same periods is bounded above by $\varepsilon(n)\left(1+\delta+\ldots+\delta^{T-(t+3)}\right)=\varepsilon(n) \sum_{l=0}^{T-(t+3)} \delta^{l}$. The first $t+2$ coordinates of $z$ determine $h_{t+2}$ which we may now take as fixed.

Let $e$ denote $E\left(v(j, 0, \widehat{f}) \mid h_{t+2}\right)$ and let $\eta=\left(\eta_{0}, \eta_{1}, \ldots\right)$ be a sequence of deviations of project values from their expected values for projects $((j, 0, \widehat{f}),(j, 1, \widehat{f}), \ldots)$. Define $(v(j, i, \widehat{f}))_{i \geq 0}$ recursively by $v(j, 0, \widehat{f})=e+\eta_{0}$ and $v(j, k, \widehat{f})=\delta v(b, k-1, \widehat{f})+\eta_{k}$ for $k \geq 1$. Also, given $\eta$ and $e$, define $S_{k}(\eta, e)=\sum_{l=0}^{k} v(j, l, \widehat{f})$. It is easy to see that $S_{k}(\eta, e)=e\left(1+\delta+\ldots+\delta^{k}\right)+$ $\eta_{1}\left(1+\delta+\ldots+\delta^{k}\right)+\eta_{2}\left(1+\delta+\ldots+\delta^{k-1}\right)+\ldots+\eta_{k}$.

As for the preliminary alternative, if $\alpha^{\prime}$ initiates $f_{i}$ at some period and $\eta_{k}$ is a realization of the deviation of $\nu\left(1, k, f_{i}\right)$ from its expected value then, given the realizations $\eta=\left(\eta_{0}, \eta_{1}, \ldots\right)$, define the hypothetical values $\left(w\left(1, i, f_{i}\right)\right)_{i \geq 0}$ recursively by $w\left(1,0, f_{i}\right)=\eta_{0}$ and $w\left(1, k, f_{i}\right)=$ $\delta w\left(1, k-1, f_{i}\right)+\eta_{k}$ for $k \geq 1$. For any $\eta, \sum_{l=0}^{k} w\left(1, l, f_{i}\right)=S_{k}(\eta, 0)$. So, for any $\eta$ and $k$, $S_{k}(\eta, e)-S_{k}(\eta, 0)$ equals $e \sum_{l=0}^{k} \delta^{l}$.

Now for an arbitrary $z=\left(z_{1}, \ldots, z_{T}\right)$ and given the equilibrium plan $a$, some subset of the

\footnotetext{
${ }^{17}$ So for example $(j, 0, \widehat{f})$ is the immediate successor in branch $j$ of the branch $j$ leading project of $\widehat{f}$. Note that the leading project of branch $j$ may be $(0, \widehat{f})$.
} 
coordinates of $z$ will be the deviations $\left(\eta_{0}, \ldots, \eta_{\tau}\right)$ for the projects in branch $j$ of $\widehat{f}$. Since $\alpha^{\prime}$ undertakes projects in $f_{j}$ if and only if $a$ undertakes projects in branch $j$ of $\widehat{f}$, the deviations for the projects undertaken by $\alpha^{\prime}$ in $f_{j}$ will be $\left(\eta_{0}, \ldots, \eta_{\tau}\right)$ when $z$ obtains. Hence given $z$ and $\varepsilon(n)$, the difference between the values delivered by $a$ from $t+3$ to $T$ and the hypothetical values delivered by $\alpha^{\prime}$ is indeed bounded above by $\varepsilon(n) \sum_{l=0}^{k} \delta^{l}$.

II. To conclude we assume that $\beta=1$ or $t \in\{T-1, T\}$ and show that initiating a new field rather undertaking a feasible project in an existing field with positive expected value cannot increase the total expected value of research. Observe first that with $\beta=1$ or $t \in\{T-1, T\}$ it is impossible to initiate a field $f$ at $t$ and then undertake projects in more than one distinct branch of $f$. Now consider plans that begin by selecting a project with expected value $v$, that always select from only one branch of any given field, and that initiate a new field immediately following the selection of a negative value project. It is easy to confirm that if $v>0$ then for any integer $n>0$ the distribution of values for the $n$th selection of such a plan will first order stochastically dominate a plan that begins a project with $v=0$. Given that the only feasible choices in any $f$ must be drawn from a single branch, total expected value is maximized by selecting the project with the highest expected value.

Uniform cutoff lemma If fixed dispersion holds and $\delta<1$, then the following two conditions are equivalent:

(1) there exists a cutoff $c>0$ such that for all $T$, all $t \leq T-2$, and all $h_{t-1}$ such that the highest value leading project has value in $(0, c)$ then any equilibrium plan underinvests in new fields following $h_{t-1}$,

(2) the number of branches in each field is greater than or equal to 2.

Proof of uniform cutoff lemma. The only change needed in the proof of the original cutoff lemma is to observe that if $\delta<1$ then the maximum difference between the expected value of research from $t+3$ to $T$ in the equilibrium and alternative strategies, $\varepsilon(1+\delta+\ldots+$ $\left.\delta^{T-(t+3)}\right)$, converges to $\frac{\varepsilon}{1-\delta}$ as $T \rightarrow \infty$. Hence by setting $c$ small enough, the gain to the alternative in periods $t$ through $t+2$ will be larger than that the equilibrium's strategy's advantage from $t+3$ to $T$.

Proof of Proposition 4. Suppose $\beta \geq 2$. We will show that for any $\varepsilon>0$ and equilibrium sequence $\left(a_{1}, a_{2}, \ldots\right)$ there exists a $T>0$ such that the probability that $\left(a_{1}, \ldots, a_{i}\right)$ underinvests in new fields at some date is greater than $1-\varepsilon$ for all $i \geq T$. Observe first that for any $c>0$ our density assumption implies that the conditional probability, given that $a_{i}$ initiates a new field $f$ at $t$, of the event described of the following three conditions is strictly positive: (1) $v(0, f)>0(2)$ in each period $t+i, i=1, \ldots, \beta-1$, the project $(i, 1, f)$ is undertaken and $v(i, 1, f)<0$ and (3) in period $t+\beta$ the project $(\beta, 1, f)$ is undertaken and $v(\beta, 1, f) \in(0, c)$. Given this observation, it is sufficient to show that any equilibrium sequence will initiate unboundedly many fields with high probability and hence eventually initiate a field $\widehat{f}$ at some $\widehat{t}$ such that $(0, \widehat{f})$ and its successors satisfy (1)-(3), which (if we choose $c$ to be sufficiently small) will imply underinvestment at date $\hat{t}+\beta+1$. More precisely we show that (I) for any integer $x>0$ and any $\varepsilon>0$ there is a $\widehat{T}$ such that, for $i \geq \widehat{T},\left(a_{1}, \ldots, a_{i}\right)$ initiates at least $x$ fields with probability at least $1-\varepsilon$, and (II) there is a $c>0$ such that if $t \leq i-2$ and $h_{t-1}$ is such that there is only one leading project $(b, j, f)$ 
with $E\left(v(b, j, f) \mid h_{t-1}\right)>0$ and $E\left(v(b, j, f) \mid h_{t-1}\right) \leq c$ then there is an alternative plan that initiates a new field at $t$ following $h_{t-1}$ that increases the total expected value of research.

I. It is sufficient to show that for any $\varepsilon>0$ there is a $t^{\prime}>0$ such that for any $f$ the probability that at least one of the numbers $v(b, 0, f), \ldots, v\left(b, t^{\prime}, f\right)$ is negative is greater than $1-\varepsilon$, since then unboundedly many fields will be initiated with high probability (even for equilibria that do not initiate new fields when only projects with expected value 0 are available). This conclusion will be an application of the martingale convergence theorem (see Williams (1991)).

Since $\delta<1$, the sequence of random variables $\left\langle V_{j}\right\rangle_{j=0}^{\infty}$, where $V_{j}=v(b, j, f)$ if $(v(b, 0, f)$, $v(b, 1, f), \ldots, v(b, j, f)) \geq 0$ and $V_{j}=v(b, i, f)$ where $i=\min \{k: v(b, k, f)<0\}$ otherwise, forms a supermartingale. If the sequence $\left\langle V_{j}\right\rangle$ were uniformly bounded in $L^{1}$ (i.e., $\exists K \in \mathbb{R}$ such that $E\left|V_{j}\right|<K$ for all $j \geq 0$ ) then the martingale convergence theorem would imply that, for a.e. $\omega, V_{j}(\omega)$ converges to a finite number as $j \rightarrow \infty$. Given fixed dispersion it would then be that for a.e. $\omega$ there is a $j$ such that $V_{j}(\omega)<0$. Now given that $E V_{j} \leq 0$ for $j \geq 0,\left\langle V_{j}\right\rangle$ would be uniformly bounded in $L_{1}$ if there were a $K \in \mathbb{R}$ such that, for $j \geq 0$, $V_{j} \geq K$ a.s. But it could be that there is no lower bound $K$ which, given our maintained assumptions on $g$, occurs only when $g(v)>0$ for all $v<0$.

To remedy this problem we adapt the $g_{e}$ densities so that the resulting sequence of random variables still forms a supermartingale, is uniformly bounded, and for positive values shares the same probabilities as $\left\langle V_{j}\right\rangle_{j=0}^{\infty}$. Assuming henceforth that $g(v)>0$ for all $v<0$, fix some $\underline{v}<0$ and $\bar{v}>0$ and pick $\mu_{1}>0$ so that $g(v) \geq \mu_{1}$ for all $v \in[\underline{v}, 0]$. Then given $e \in[0, \bar{v}]$ set $\mu_{2}(e)>0$ and $\widetilde{v}<\underline{v}$ so that

$$
\int_{\underline{v} / 2}^{0} v \mu_{1} d v+\int_{-\infty}^{\widetilde{v}} v g_{e}(v) d v=\int_{\underline{v}}^{\underline{v} / 2} v \mu_{2}(e) d v .
$$

It is readily confirmed that for the function $\widehat{g}_{e}$ defined by

$$
\widehat{g}_{e}(v)= \begin{cases}0 & \text { for } v \in(-\infty, \widetilde{v}] \\ g_{e}(v)+\mu_{2}(e) & \text { for } v \in[\underline{v}, \underline{v} / 2) \\ g_{e}(v)-\mu_{1} & \text { for } v \in[\underline{v} / 2,0] \\ g_{e}(v) & \text { otherwise }\end{cases}
$$

we have $\int_{-\infty}^{\infty} v \widehat{g}_{e}(v) d v=\int_{-\infty}^{\infty} v g_{e}(v) d v=e$. Notice that if $\widetilde{v}$ is sufficiently small and hence $\int_{-\infty}^{\widetilde{v}} v g_{e}(v) d v$ is sufficiently near $0,(3)$ implies $\mu_{2}(e)<\mu_{1}$. We may in fact choose one $\widetilde{v}$ such that, for all $e \in[0, \bar{v}], \mu_{2}(e)<\mu_{1}$. To see this, observe that for any $e \geq 0$

$$
\int_{-\infty}^{\widetilde{v}} v g_{e} d v=\int_{-\infty}^{\widetilde{v}} v g(v-e) d v=\int_{-\infty}^{\widetilde{v}-e}(v+e) g(v) d v \geq \int_{-\infty}^{\widetilde{v}-e} v g(v) d v \geq \int_{-\infty}^{\widetilde{v}} v g(v) d v
$$

with the second equality due to a change of variables (from $v-e$ to $v$ ). So set $\widetilde{v}$ small enough that the $\mu_{2}$ that satisfies $\int_{\underline{v} / 2}^{0} v \mu_{1} d v+\int_{-\infty}^{\widetilde{v}} v g(v) d v=\int_{\underline{v}}^{\underline{v} / 2} v \mu_{2} d v$ is such that $\mu_{2}<\mu_{1}$. Then (4) implies that $\mu_{2}(e) \leq \mu_{2}$ for any $e \in[0, \bar{v}]$. Given that $\mu_{2}(e)<\mu_{1}$, we have $\int_{-\infty}^{\infty} \widehat{g}_{e}(v) d v<1$ and so $\widehat{g}_{e}$ is not a density. We therefore define $\widetilde{g}_{e}$ by $\widetilde{g}_{e}(v)=\alpha \widehat{g}_{e}(v)$ for $v<0$ and $\widetilde{g}_{e}(v)=\widehat{g}_{e}(v)$ for $v \geq 0$, where $\alpha>1$ is set by $\int_{-\infty}^{\infty} \alpha \widehat{g}_{e}(v) d v=1$. Since 
$\int_{-\infty}^{\infty} v \widehat{g}_{e}(v) d v=e, \int_{-\infty}^{\infty} v \widetilde{g}_{e}(v) d v<e$.

For $e>\bar{v}$, set $\widetilde{v}<0$ and define $\widetilde{g}_{e}$ by

$$
\widetilde{g}_{e}(v)= \begin{cases}0 & \text { for } v \in(-\infty, \widetilde{v}] \\ \alpha g_{e}(v) & \text { for } v \in(\widetilde{v}, 0) \\ g_{e}(v) & \text { for } v \in[0, \infty)\end{cases}
$$

where $\alpha>1$ is chosen so that $\int_{-\infty}^{\infty} \widetilde{g}_{e}(v) d v=1$. While $\int_{-\infty}^{\infty} v \widetilde{g}_{e}(v) d v>e$, it is the case that $\int_{-\infty}^{\infty} v \widetilde{g}_{e}(v) d v \leq e-\int_{-\infty}^{\widetilde{v}} v g_{e} d v \leq e-\int_{-\infty}^{\widetilde{v}} v g d v$, with the second inequality due to (4). If $\widetilde{v}$ is sufficiently small then, since $\delta<1, e-\int_{-\infty}^{\widetilde{v}} v g d v \leq \frac{1}{\delta} e$ for all $e>\bar{v}$.

Now replace each $g_{e}, e \geq 0$, in the fixed dispersion model with $\widetilde{g}_{e}$ where $\widetilde{v}$ may be any fixed number smaller than the values identified in the last two paragraphs. The $\widetilde{g}_{e}$ define a new probability $\widetilde{P}$ on $\Omega$ and thus the random variables $\left\langle\widetilde{V}_{j}\right\rangle_{j=0}^{\infty}$. Given that, for all $e \geq 0, \int_{-\infty}^{\infty} v \widetilde{g}_{e}(v) d v \leq \frac{1}{\delta} e,\left\langle\widetilde{V}_{j}\right\rangle_{j=0}^{\infty}$ forms a supermartingale. Since for any $k \geq 0$ and any measurable set of sequences of the form $\mathcal{V}_{k}=\left\{\left\langle v_{j}\right\rangle_{j=0}^{\infty}: v_{j} \geq 0, j \leq k\right\}, P\left(\left\langle V_{j}\right\rangle_{j=0}^{\infty} \in \mathcal{V}_{k}\right)=$ $\widetilde{P}\left(\left\langle\widetilde{V}_{j}\right\rangle_{j=0}^{\infty} \in \mathcal{V}_{k}\right)$, we have $P\left(V_{k}<0\right)=\widetilde{P}\left(\widetilde{V}_{k}<0\right)$ and $P\left(V_{j}<0\right.$ for some $\left.j \geq 0\right)=\widetilde{P}\left(\widetilde{V}_{j}<0\right.$ for some $j \geq 0)$. Hence part I is concluded if the martingale convergence theorem applies to $\left\langle\widetilde{V}_{j}\right\rangle$. Since each $\widetilde{V}_{j} \geq \widetilde{v}$ a.s. (and since $E \widetilde{V}_{j} \leq 0$ for $j \geq 0$ ), $\left\langle\widehat{V}_{j}\right\rangle$ is uniformly bounded in $L^{1}$ and so the martingale convergence theorem does apply.

II. This is an application of the uniform cutoff lemma.

Part II of the proof of the Cutoff Lemma shows that when $\beta=1$ equilibria cannot underinvest in new fields (the 'only if' half of Proposition 4).

Proof of Proposition 5. Let $F(\cdot)$ denote the distribution function of $v(\pi)$ and let $H(v, \cdot)$ denote the conditional distribution function of $Z$ given $v(\pi)=v$. Let $t$ be the date at which the choice between $\pi$ and $\widetilde{\pi}$ is made. If $\widetilde{\pi}$ is chosen and $\delta v(\widetilde{\pi}) \leq \bar{v}$ then the value achieved at $t+1$ is $\bar{v}$. So the expected value at $t+1$ when $\tilde{\pi}$ is chosen at $t$ equals

$$
\int_{-\infty}^{\infty}\left(\int_{Z>(1 / \delta) \bar{v}-v} \delta(v+Z) d H(v, Z)+\int_{Z \leq(1 / \delta) \bar{v}-v} \bar{v} d H(v, Z)\right) d F(v) .
$$

Observe that for any $v$,

$$
\int_{Z>(1 / \delta) \bar{v}-v} \delta(v+Z) d H(v, Z)+\int_{Z \leq(1 / \delta) \bar{v}-v} \bar{v} d H(v, Z) \geq \int_{-\infty}^{\infty} \delta(v+Z) d H(v, Z)=\delta v,
$$

where the equality follows from (1) in the definition of mean-preserving spread, and

$$
\int_{Z>(1 / \delta) \bar{v}-v} \delta(v+Z) d H(v, Z)+\int_{Z \leq(1 / \delta) \bar{v}-v} \bar{v} d H(v, Z) \geq \bar{v} .
$$

Hence

$$
\int_{Z>(1 / \delta) \bar{v}-v} \delta(v+Z) d H(v, Z)+\int_{Z \leq(1 / \delta) \bar{v}-v} \bar{v} d H(v, Z) \geq \max [\delta v, \bar{v}] .
$$


and

$$
\int_{-\infty}^{\infty}\left(\int_{Z>(1 / \delta) \bar{v}-v} \delta(v+Z) d H(v, Z)+\int_{Z \leq(1 / \delta) \bar{v}-v} \bar{v} d H(v, Z)\right) d F(v) \geq \int_{-\infty}^{\infty} \max [\delta v, \bar{v}] d F(v) .
$$

Since if $\pi$ is undertaken at $t$ the expected value $\int_{-\infty}^{\infty} \max [\delta v, \bar{v}] d F(v)$ is achieved at $t+1$, the sum of the expected values of research at $t$ and $t+1$ is at least as great if $\widetilde{\pi}$ rather than $\pi$ is undertaken. If $\tilde{\pi}$ 's greater risk is nontrivial relative to $\bar{v}$ then either the inequality in (5) or in (6) is strict for a positive measure set of $v$ and hence the inequality in (7) is strict.

Proof of Proposition 7. For any $\bar{v}>0$, it is a positive probability event for each country $i=1, \ldots, n-1$ at its first move to draw a root project $f_{i}$ with $0<v\left(0, f_{i}\right)<\bar{v}$, and for country $n$ to draw only negative-value root projects at every period it chooses through $t-1$. Then under autarchy country $n$ will initiate at the first period $\tau \geq t$ at which $n$ chooses but will not initiate at $\tau$ if full communication begins at $t$. Set $\bar{v}$ to satisfy $\delta \int_{\bar{v}}^{\infty}(v-\bar{v}) g(v) d v>\bar{v}>0$. Since the Proposition's restriction on $t$ means that $n$ chooses at least twice from the dates $\{t, \ldots, T\}$, Proposition 1 implies that the total expected value of research will be higher if country $n$ initiates at $\tau$.

Proof of Proposition 8. I. Suppose $\delta>1$. Given a citations equilibrium, with probability 1 every root project $\pi$ undertaken has $v(\pi) \neq 0$ and we exclude any state where this condition does not obtain. In the citations equilibrium, agent $T$ will pick a $(b, i, f)$ such that $v(b, i-1, f)$ is maximal among the leading projects available at $T$, assuming that one of these is positive. If this assumption does not hold - when each root project $\pi$ undertaken before $T$ has $v(\pi)<0$ - then $T$ will initiate. Since therefore agent $T-1$ earns one citation if and only if he chooses a $\pi$ such that $v(\pi)$ is maximal among the leading projects at $T$, agent $T-1$ will choose a $(b, i, f)$ such that $v(b, i-1, f)$ is maximal among the leading projects available at $T-1$, again assuming that one such value is positive. By induction, each $i$ such that $1<i<T-1$ will also choose a $(b, i, f)$ such that $v(b, i-1, f)$ is maximal among the leading projects available at $i-1$ when one such value is positive.

II. The $\delta=1$ case is covered in the text, where implicitly we ignored states where projects had 0 value.

III. Suppose that $\delta<1$ and that (i) and (ii) hold. Consider a citations equilibrium and history where the first root project $(0, f)$ revealed to have $v(0, f)>0$ leaves an odd number of periods remaining, which we number $1, \ldots, T$. Since the argument given for the $\delta>1$ case applies to $\delta<1$ models that satisfy (i) and (ii) and have $\beta=1$, assume that $\beta=2$. We show that the on equilibrium path of any citations equilibrium, each $t \in\{1, \ldots, T-1\}$ undertakes a project in $f$ and, if $1, \ldots, t-1$ have chosen from $f$ and the two projects available at $t$ in $f$ are $(1, i, f)$ and $(2, j, f)$ then $t$ will (1) choose the project with the smaller of $i$ and $j$ if $i \neq j$ and $(2)$ choose $(1, i, f)$ and $(2, j, f)$ each with probability $\frac{1}{2}$ if $i=j$. Since $f$ is the first field with a positive-value root project, these actions can be taken in a value-seeking equilibrium, regardless of the realization of the randomization. Since most of $t$ 's off-the-equilibrium-path actions will not be relevant to the proof, we leave them unspecified. It is straightforward to argue by induction that for the histories where we have not indicated any action there are actions consistent with a citations equilibrium.

Agent $T$ must take only value-maximizing actions at any $h_{T-1}$ in a citations equilibrium. In particular, if agents $1, \ldots, T-1$ do not initiate a new field and $(1, i, f)$ and $(2, j, f)$ are 
available at $T$, then $T$ undertakes the project with the smaller of $i$ and $j$ if $i \neq j$ and otherwise chooses each with probability $\frac{1}{2}$. Note that when $1, \ldots, T-1$ adopt the strategies we have posited, $i=j$ (since $T-1$ is even) and $T$ will mix. We claim that, for $t=1, \ldots, T-1$, if agents $1, \ldots, t-1$ follow the posited equilibrium actions then so will $t$. To argue by induction, suppose the claim is true for $t+1, \ldots, T$ and let $\left.\left\{(b, 1, f), \ldots,(b, i, f),\left(b^{\prime}, 1, f\right), \ldots,\left(b^{\prime}, j, f\right)\right)\right\}$ be the set of projects undertaken by $1, \ldots, t-1$, where $b \neq b^{\prime}$ and $i+j=t-1$.

Suppose first that $t$ is an even integer between 2 and $T-1$ which implies that $i=$ $j+1$. Then $t$ earns at least $\frac{1}{2}(T-t)$ expected citations with the posited equilibrium action of undertaking $\left(b^{\prime}, j+1, f\right)$. If $t$ instead undertakes $(b, i+1, f)$ then $t$ earns at most $\frac{1}{2}(T-t-2)$ expected citations. The only other action available to $t$ is to initiate a new field $f^{\prime}$. Assumption (i) then implies that, with probability greater than $\frac{1}{2}, v\left(0, f^{\prime}\right)<v(b, T, f)$ and hence $t$ earns no citations. So $t$ 's expected number of citations is less than $\frac{1}{2}(T-t)$ if $t$ initiates a new field.

To conclude, let $t$ be an odd integer between 1 and $T-2$ which implies that $i=j$. Then undertaking either $(b, i+1, f)$ or $\left(b^{\prime}, j+1, f\right)$ earns $\frac{1}{2}(T-t-1)$ expected citations. So if $t$ does not initiate a new field, $t$ will choose $(b, i+1, f)$ and $\left(b^{\prime}, j+1, f\right)$ each with probability $\frac{1}{2}$. To show that $t$ 's expected number of citations if $t$ initiates a new field $f^{\prime}$ is less than $\frac{1}{2}(T-t-1)$, it is sufficient to show that in the event $v\left(0, f^{\prime}\right)>v(v, T, f)$, which has probability less than $\frac{1}{2}$, at least one agent from $t+1$ through $T$ does not undertake a project in $f^{\prime}$. Suppose to the contrary that all of the agents in $S=\{t+1, \ldots, T-1\}$ undertake projects in $f^{\prime}$. Since $|S|$ is odd at least one agent $\widehat{t}$ in $S$ must choose a $\left(b, i, f^{\prime}\right)$ such that $i>j$ for each $\left(b^{\prime}, j, f^{\prime}\right)$ that is chosen by an agent in $S \backslash\{\widehat{t}\}$. Given that $\delta<1$, agent $T$ will not choose from branch $b$ of $f^{\prime}$ and so there can be no $\widetilde{t}>\widehat{t}$ who chooses from branch $b$. Hence $\widehat{t}$ earns 0 citations and would increase his expected number of citations by initiating a new field $f^{\prime \prime}$.

\section{References}

[1] Banerjee, A., 1992, 'A simple model of herd behavior,' Quarterly Journal of Economics 107: 797-818.

[2] Bikhchandani, S., Hirshleifer, D. and Welch, I., 1992, 'A theory of fads, fashion, custom, and change as informational cascades,' Journal of Political Economy 100: 992-1026.

[3] Dasgupta, P. and David, P., 1994, 'Toward a new economics of science,' Research Policy 23: $487-521$.

[4] Dasgupta, P. and Maskin, E., 1987, 'The simple economics of research portfolios,' The Economic Journal 97: 581-595.

[5] Jovanovic, B. and Nyarko, Y., 1996, 'Learning by doing and the choice of technology,' Econometrica 64: 1299-1310.

[6] Hoddeson, L., Brown, B., Riordan, M., and Dresden, M. (eds.), 1997, The Rise of the Standard Model: A History of Particle Physics from 1964 to 1979, Cambridge University Press, Cambridge. 
[7] Hong, L., and Page, S., 2004, 'Groups of diverse problem solvers can outperform groups of high-ability problem solvers,' Proceedings of the National Academy of Sciences 101: $16385-16389$.

[8] Kuhn, T., 1962, The Structure of Scientific Revolutions, University of Chicago Press: Chicago.

[9] Kuznets, S., 1930, Secular Movements in Production and Prices, Houghton-Mifflin, Boston.

[10] McCall, J., 1970, 'Economics of information and job search,' Quarterly Journal of Economics 84: 113-126.

[11] Ne'eman, Y., 1982, 'Gauge-theory ghosts and ghost-gauge theories,' in Differential Geometric Methods in Mathematical Physics, Clausthal 1980 (H.-D. Doebner, S. Andersson, and H. Petry, eds.), Lecture notes in mathematics 905, Springer, Berlin, p. 241-259.

[12] Polyakov, A., 1997, 'A view from the island,' in The Rise of the Standard Model: A History of Particle Physics from 1964 to 1979 (Hoddeson et al., eds.), Cambridge University Press, Cambridge, p. 243-249.

[13] Rothschild, M., and Stiglitz, J., 1970, 'Increasing risk: I. a definition,' Journal of Economic Theory 2: 225-243.

[14] Schumpeter, J., 1911, The Theory of Economic Development, Harvard University Press, Cambridge MA, 1934.

[15] 't Hooft, G., 1999, 'When was asymptotic freedom discovered? or The rehabilitation of quantum field theory,' Nuclear Physics B 74: 413-425.

[16] Williams, D., 1991, Probability with Martingales, Cambridge University Press, Cambridge. 